Homology, Homotopy and Applications, vol.17(1), 2015, pp.35-66

\title{
SCANNING FOR ORIENTED CONFIGURATION SPACES
}

\author{
JEREMY MILLER AND MARTIN PALMER
}

(communicated by Dev P. Sinha)

\begin{abstract}
In $[$ Pal13 $]$ the second author proved that the sequence of "oriented" configuration spaces on an open connected manifold exhibits homological stability as the number of particles goes to infinity. To complement that result we identify the corresponding limiting space, up to homology equivalence, as a certain explicit double cover of a section space. Along the way we also prove that the scanning map of [McD75] for unordered configuration spaces is acyclic in the limit.
\end{abstract}

\section{Introduction}

There are many interesting examples of families of spaces $\left\{Y_{k}\right\}$ whose homology groups $H_{i}\left(Y_{k}\right)$ are independent of $k$ for $k \gg i$. Examples include the classifying spaces of general linear groups [Qui73, Cha80], mapping class groups [Har85, Wah08], automorphism groups of free groups [Hat95, HV98, HW10], and unordered configuration spaces of particles in an open connected manifold [McD75, Seg79]. In many of these cases (see, for example, [McD75, MW07, Gal11]) one can find a computationally more tractable space $Z$ that is homology equivalent to the limiting space $\operatorname{hocolim}_{k}\left(Y_{k}\right)$. In [Pal13] the second author proved homological stability for oriented configuration spaces, and the purpose of this paper is to describe the corresponding limiting space.

Oriented configuration spaces are natural generalizations of the classifying spaces of the alternating groups. One possible motivation for their study was given in [GKY], where it was shown that homological stability for oriented configuration spaces implies stability for the homotopy groups of spaces of positive and negative particles. We will also describe an application of these ideas to the study of the homology of the spaces appearing in the generalized Snaith splitting of [Böd87].

Background. Before we state the results of this paper and of [Pal13], we first fix some notation and review some classical theorems regarding configuration spaces of unordered particles. Let $F_{k}(M):=M^{k} \backslash \Delta_{f}$, where $\Delta_{f}$ is the fat diagonal. Define

Received June 28, 2013, revised March 17, 2014, June 17, 2014, September 12, 2014; published on April 9, 2015.

2010 Mathematics Subject Classification: 55R80, 55R65, 57N65.

Key words and phrases: oriented configuration space, alternating group, scanning, homology fibration, local coefficients, group completion.

Article available at http://dx.doi.org/10.4310/HHA.2015.v17.n1.a2

Copyright (C) 2015, Jeremy Miller and Martin Palmer. Some rights reserved under Creative Commons license CC BY-NC. Permission to copy for private use granted. 
$C_{k}(M)$ to be the quotient of $F_{k}(M)$ by the action of the symmetric group $\Sigma_{k}$, and define $C_{k}^{+}(M)$ to be the quotient by the action of the alternating group $A_{k}$. We call these spaces, respectively, the configuration spaces of ordered, unordered and oriented collections of points in $M$.

Throughout, we require that the manifold $M$ be connected and of dimension at least 2. We say that a manifold admits boundary if it is the interior of a (not necessarily compact) manifold with (not necessarily compact) non-empty boundary. For such manifolds, Segal proved in [Seg79] the following theorem.

Theorem 1.1 ([Seg79, Proposition A.1]). If $M$ is a manifold admitting boundary, there is a map $t: C_{k}(M) \rightarrow C_{k+1}(M)$ which induces an isomorphism on homology for $* \leqslant k / 2$.

We call the map $t$ the "stabilization map." Roughly, it involves moving all the particles away from the boundary and then adding a new particle near the boundary; see $\S 3$ for precise definitions. No such map exists for closed manifolds, and, in fact, homological stability fails for closed manifolds in general. ${ }^{1}$

Let $\pi: \dot{T} M \rightarrow M$ denote the fiberwise one-point compactification of the tangent bundle of $M$, and let $\Gamma(M)$ denote the space of compactly-supported sections of this bundle. There is a natural bijection $\pi_{0}(\Gamma(M)) \rightarrow \mathbb{Z}$ given by the degree of a compactly-supported section, whose definition we recall in $\S 3.1$. For $k \in \mathbb{Z}$ we denote the path component of $\Gamma(M)$ consisting of degree- $k$ sections by $\Gamma_{k}(M)$. In [McD75] McDuff defined a scanning map $s: C_{k}(M) \rightarrow \Gamma_{k}(M)$ and proved the following two theorems.

Theorem 1.2 ([McD75, Theorem 1.2]). If $M$ is a manifold admitting boundary, the scanning maps $s: C_{k}(M) \rightarrow \Gamma_{k}(M)$ induce an isomorphism $H_{*}\left(C_{\infty}(M) ; \mathbb{Z}\right) \rightarrow$ $H_{*}\left(\Gamma_{\infty}(M) ; \mathbb{Z}\right)$.

Here $C_{\infty}(M)$ denotes the homotopy colimit of the maps

$$
\cdots \rightarrow C_{k}(M) \rightarrow C_{k+1}(M) \rightarrow \cdots
$$

from Theorem 1.1, and $\Gamma_{\infty}(M)$ denotes the homotopy colimit of analogous "stabilization" maps for the path components $\Gamma_{k}(M)$ of $\Gamma(M)$.

Theorem 1.3 ([McD75, Theorem 1.1]). The scanning map $s: C_{k}(M) \rightarrow \Gamma_{k}(M)$ induces an isomorphism on homology in the same range $(* \leqslant k / 2)$ as the map $t: C_{k}(M \backslash p t) \rightarrow C_{k+1}(M \backslash p t)$.

When the manifold $M$ admits boundary, the spaces $\Gamma_{k}(M)$ are all homotopy equivalent (see $\S 3.3$ ), so this provides a limiting space $Z$ for the sequence $\left\{C_{k}(M)\right\}$. When $M$ is closed, we just have two sequences $\left\{C_{k}(M)\right\}$ and $\left\{\Gamma_{k}(M)\right\}$ which become better and better approximations of each other as $k \rightarrow \infty$.

${ }^{1}$ For example, from the presentation of $\pi_{1}\left(C_{k}\left(S^{2}\right)\right)$ in $\left[\right.$ FVB62] we have $H_{1}\left(C_{k}\left(S^{2}\right) ; \mathbb{Z}\right) \cong$ $\mathbb{Z} /(2 k-2)$, which is not stable as $k \rightarrow \infty$. Homological stability does, however, hold rationally [Chu12, RW13] and for mod-2 coefficients [ML88, BCT89, RW13]. See also [BM14] and [CP14] for more stability results for the torsion in the homology of unordered configuration spaces on closed manifolds. 
Oriented configuration spaces. The analogue of Theorem 1.1 for oriented configuration spaces is the following:

Theorem 1.4 ([Pal13]). Let $M$ be a manifold admitting boundary. Then there is a map $t^{+}: C_{k}^{+}(M) \rightarrow C_{k+1}^{+}(M)$ which induces an isomorphism on homology for $* \leqslant$ $(k-5) / 3$ and a surjection for $* \leqslant(k-2) / 3$.

The goal of this paper is to provide analogues of Theorem 1.2 and Theorem 1.3 for oriented configuration spaces. For $k \geqslant 2$, the scanning map $s: C_{k}(M) \rightarrow \Gamma_{k}(M)$ induces an isomorphism on $H^{1}(-; \mathbb{Z} / 2)$, by Theorem 1.3 above and the universal coefficient theorem. Cohomology with mod-2 coefficients classifies double covers of a space, so this says that any double cover of $C_{k}(M)$ is the pullback of a (unique) double cover of $\Gamma_{k}(M)$. In particular, $C_{k}^{+}(M)$ fits into a pullback square:

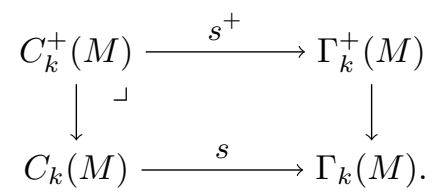

There is an alternative, more geometric description of the associated double cover $\Gamma_{k}^{+}(M) \rightarrow \Gamma_{k}(M)$ which is described in $\S 3.6$. Our analogue of Theorem 1.3 is:

Theorem A. The lifted scanning map $s^{+}: C_{k}^{+}(M) \rightarrow \Gamma_{k}^{+}(M)$ induces an isomorphism on homology in the range $* \leqslant(k-5) / 3$ and a surjection for $* \leqslant(k-2) / 3$.

Again, when the manifold $M$ admits boundary the spaces $\Gamma_{k}^{+}(M)$ are all homotopy equivalent, so this provides a limiting space $Z$ for the sequence $\left\{C_{n}^{+}(M)\right\}$.

Note that unlike configuration spaces of unordered particles, or more generally configuration spaces with summable labels [Sal01], oriented configuration spaces are not local: to determine a point in $C_{k}^{+}(M)$ one needs more than the information attached to each point in the configuration. Nevertheless, they still exhibit homological stability, and we can still, via Theorem A, describe a limiting space.

To prove Theorem A we first prove the following strengthening of Theorem 1.2:

Theorem B. If $M$ is a manifold admitting boundary, the scanning map in the limit $s: C_{\infty}(M) \rightarrow \Gamma_{\infty}(M)$ is acyclic.

In $\S 3$ we define concrete models for configuration and section spaces so that the stabilization and scanning maps commute on the nose. The scanning maps therefore induce a well-defined map from the mapping telescope of the sequence of stabilization maps for configuration spaces to the mapping telescope of the sequence of stabilization maps for section spaces. This is the map $s$ referred to in the above theorem.

This theorem, combined with the stability result from [Pal13], directly implies Theorem A in the case of manifolds admitting boundary (see Corollary 5.9). In $\S 6$ we show how to extend Theorem A to closed manifolds.

We emphasize that our method of deducing Theorem A from Theorem B uses homological stability for oriented configuration spaces with untwisted coefficients, so we cannot deduce that the lifted scanning map $s^{+}: C_{k}^{+}(M) \rightarrow \Gamma_{k}^{+}(M)$ is acyclic in a range of degrees depending on $k$. We only know that it is a homology equivalence in a 
range, and acyclic in all homological degrees when $k \rightarrow \infty$. The question of acyclicity in a range before taking the limit is not pursued here.

To prove Theorem B we need the notion of an abelian homology fibration, a twisted version of the homology fibration criterion of [McD75] and a corollary of a twisted version of the group-completion theorem. The full details of all this are worked out in [MP14], following the ideas of [McD75] and [MS75] and using a result of [RW13]. The particular definitions and results which are needed for the present paper are recalled in $\S 2$.

Twisted and abelian homology equivalences. We now fix terminology for some different notions of homology equivalence. Let $f: X \rightarrow Y$ be a continuous map of spaces, and let $\mathcal{F}$ denote a local coefficient system on $Y$ - this can be thought of as a functor $\pi(Y) \rightarrow \mathrm{Ab}$ from the fundamental groupoid of $Y$ to the category of abelian groups or as a bundle of abelian groups over $Y$. It is called abelian if, in the bundle viewpoint, the monodromy of any fiber around a commutator loop is trivial. In the functor viewpoint this says that for each $y \in Y$ the homomorphism $\pi_{1}(Y, y)=\pi(Y)(y, y) \rightarrow \operatorname{Aut}_{\mathrm{Ab}}(\mathcal{F}(y))$ factors through an abelian group.

The map $f$ is called acyclic, or a twisted homology equivalence, if it induces isomorphisms $H_{*}\left(X ; f^{*} \mathcal{F}\right) \rightarrow H_{*}(Y ; \mathcal{F})$ for all local coefficient systems $\mathcal{F}$ on $Y$. It is called an abelian homology equivalence if it induces isomorphisms for all abelian local coefficient systems on $Y$, and it is called a trivial homology equivalence, or just a homology equivalence, if it induces isomorphisms for the trivial coefficient system $\mathbb{Z}$ (i.e., the trivial bundle $\mathbb{Z} \times Y \rightarrow Y$ ). An alternative characterization (see [Ber82, Proposition 4.3]) of acyclicity of $f$ is that $\widetilde{H}_{*}($ hofib $(f) ; \mathbb{Z})=0$ in all degrees (where $\mathbb{Z}$ is a trivial coefficient system). From this it follows that the homotopy pullback of an acyclic map is acyclic. In particular, in diagram (1.1), once $k \rightarrow \infty$, acyclicity of $s$ will imply acyclicity of $s^{+}$.

The sign representation. One can rephrase results about oriented configuration spaces in terms of homology of unordered configuration spaces with certain twisted coefficients. Let $\rho: \pi_{1}\left(C_{k}(M)\right) \rightarrow \mathbb{Z} / 2$ be the composition of the natural map $\pi_{1}\left(C_{k}(M)\right) \rightarrow \Sigma_{k}$ and the sign homomorphism $\Sigma_{k} \rightarrow \mathbb{Z} / 2$. For a ring $R$ the group$\operatorname{ring} R[\mathbb{Z} / 2]$ is given the structure of an $R\left[\pi_{1}\left(C_{k}(M)\right)\right]$-module by the homomorphism $\rho$. By the definition of local homology, or a trivial application of the Serre spectral sequence to the fibration $S^{0} \rightarrow C_{k}^{+}(M) \rightarrow C_{k}(M)$, we have an isomorphism:

$$
H_{*}\left(C_{k}^{+}(M) ; R\right) \cong H_{*}\left(C_{k}(M) ; R[\mathbb{Z} / 2]\right) .
$$

When 2 is invertible in $R$, the module $R[\mathbb{Z} / 2]$ decomposes as $R \oplus R^{(-1)}$, where $\pi_{1}\left(C_{k}(M)\right)$ acts trivially on $R$, and on $R^{(-1)}$ it acts by $\rho$ and the action of $\mathbb{Z} / 2$ given by $r \mapsto-r$ (the "sign representation"). Hence we have a decomposition:

$$
H_{*}\left(C_{k}^{+}(M) ; R\right) \cong H_{*}\left(C_{k}(M) ; R\right) \oplus H_{*}\left(C_{k}(M) ; R^{(-1)}\right) .
$$

Theorem $\mathrm{B}$ allows one to study the homology of the spaces $C_{k}(M)$ with this twisted coefficient system. The groups $H_{*}\left(C_{k}(M) ; \mathbb{Z}^{(-1)}\right)$ are interesting for the following reason. Let $M$ be a closed, almost parallelizable $d$-manifold. Then for any $m>0$ the space $\operatorname{Map}_{*}\left(M, S^{d+m}\right)$ of based maps splits stably (in the sense of stable homotopy theory) into summands which are Thom spaces of vector bundles over 
$C_{k}(M \backslash p t)$ (Propositions 2 and 3 of [Böd87]; see also [BCT89]). The construction recovers the Snaith splitting [Sna74] when $M=S^{d}$. The Thom isomorphism theorem implies that the homologies of these Thom spaces are shifts of $H_{*}\left(C_{k}(M \backslash p t) ; \mathbb{Z}\right)$ or $H_{*}\left(C_{k}(M \backslash p t) ; \mathbb{Z}^{(-1)}\right)$, depending on whether or not the corresponding vector bundles are orientable. So to understand the homology of the spaces appearing in generalized Snaith splitting one needs to understand the homology of configuration spaces with sign-twisted coefficients.

Outline. We begin in $\S 2$ by recalling two important tools which we will need: we recall from [MP14] a twisted version of McDuff's homology fibration criterion (Proposition 2.3) and a corollary of a twisted version of the group-completion theorem (Proposition 2.4). In $\S 3$ we then carefully describe models for the stabilization and scanning maps that commute on the nose. In $\S 4$ we prove acyclicity of the scanning map in the limit (Theorem B) in the special case when the manifold $M$ is $\mathbb{R}^{m}$. For this we need Proposition 2.4 applied to a certain topological monoid built from configurations in $\mathbb{R}^{m}$. In $\S 5$ this special case is used to extend Theorem B to any manifold $M$ admitting boundary; this step requires the use of the abelian homology fibration criterion (Proposition 2.3). From this we deduce that the lifted scanning map is a homology equivalence in a stable range (Theorem A) when the manifold $M$ admits boundary. Finally, in $\S 6$ we show how to extend this latter result to closed manifolds and also discuss the scanning map on homology twisted by the sign representation.

\section{Acknowledgments}

We would like to thank Oscar Randal-Williams and Ulrike Tillmann for several enlightening discussions. Additionally, we thank the anonymous referee for many helpful suggestions and corrections, which have much improved this paper.

\section{An abelian homology fibration criterion and a corollary of the group-completion theorem}

In this section we review some results from [MS75], [RW13], and [MP14]. We recall the definition of an abelian homology fibration, give a criterion for recognizing these, and then state a corollary of the (twisted) group-completion theorem. In [MP14] one works with a class of local coefficient systems $\mathfrak{C}$ that is closed under taking pullbacks. Here we specialize $\mathfrak{C}$ to the class of all abelian local coefficient systems, but the results in [MP14] apply in the general situation.

Given a map $r: Y \rightarrow X$ and a point $z \in X$, we denote the homotopy fiber of $r$ over $z$ by hofib $z(r)$.

Definition 2.1. A map $r: Y \rightarrow X$ is called an abelian homology fibration if for all points $z \in X$ the natural inclusion $r^{-1}(z) \rightarrow \operatorname{hofib}_{z}(r)$ is an abelian homology equivalence. That is, if $\mathcal{F}$ is an abelian local coefficient system on $\operatorname{hofib}_{z}(r)$ and $i: r^{-1}(z) \rightarrow \operatorname{hofib}_{z}(r)$ is the natural inclusion, then $i$ induces an isomorphism:

$$
i_{*}: H_{*}\left(r^{-1}(z) ; i^{*} \mathcal{F}\right) \longrightarrow H_{*}\left(\operatorname{hofib}_{z}(r) ; \mathcal{F}\right) .
$$


To state the recognition criterion for abelian homology fibrations we need the following:

Definition 2.2. We say that a map $r: Y \rightarrow X$ is locally stalk-like over $Z \subseteq X$ if there is a basis $\mathcal{B}$ for the topology of $Z$ such that each $U \in \mathcal{B}$ is contractible and contains a point $z_{U}$ such that the inclusion $r^{-1}\left(z_{U}\right) \hookrightarrow r^{-1}(U)$ is a weak equivalence.

Note that this is a much weaker condition than requiring this inclusion to be a weak equivalence for every point in $U$.

The following is a version of McDuff's homology fibration criterion (Proposition 5.1 of [McD75]) that applies to the class of abelian local coefficient systems. It follows from Theorem 3.1 and Remark 3.2 of [MP14] with $\mathfrak{C}$ taken to be the class of all abelian local coefficient systems.

Proposition 2.3. Let $X$ be a topological space with closed filtration $\left\{X_{n}\right\}_{n \in \mathbb{N}}$, meaning that the $X_{n}$ are closed subsets of $X$ satisfying $X_{n-1} \subseteq X_{n}, X=\bigcup_{n \in \mathbb{N}} X_{n}$ and each compact subset of $X$ is contained in some $X_{n}$. Let $r: Y \rightarrow X$ be a map and assume that each $X_{n}$ and $r^{-1}\left(X_{n}\right)$ are Hausdorff. Then $r$ is an abelian homology fibration if the following three conditions are satisfied:

(i) The map $r$ is locally stalk-like over each $X_{n}$.

(ii) The restriction of $r$ to each $X_{n} \backslash X_{n-1}$ and to $X_{0}$ is an abelian homology fibration.

(iii) For each $n$ there is $X_{n-1} \subseteq U_{n} \subseteq X_{n}$, with $U_{n}$ open in $X_{n}$, and homotopies $h_{t}: U_{n} \rightarrow U_{n}$ and $H_{t}: r^{-1}\left(\bar{U}_{n}\right) \rightarrow r^{-1}\left(U_{n}\right)$ satisfying the following:

(a) $h_{0}=i d, h_{t}\left(X_{n-1}\right) \subseteq X_{n-1}, h_{1}\left(U_{n}\right) \subseteq X_{n-1}$;

(b) $H_{0}=i d, r \circ H_{t}=h_{t} \circ r$;

(c) for all $x \in U_{n}, H_{1}: r^{-1}(x) \rightarrow r^{-1}\left(h_{1}(x)\right)$ is an abelian homology equivalence.

We now discuss an application of the group-completion theorem to topological monoids. Let $\mathcal{M}$ be a topological monoid with $\pi_{0}(\mathcal{M})=\mathbb{N}$. Denote its components by $\mathcal{M}_{k}$, choose an element $m \in \mathcal{M}_{1}$, and define $\mathcal{M}_{\infty}$ to be the mapping telescope of the sequence of maps $\mathcal{M} \rightarrow \mathcal{M} \rightarrow \mathcal{M} \rightarrow \cdots$, where each map is right-multiplication by $m$.

Remark 2 of [MS75] states that there is a weak equivalence between $\mathcal{M}_{\infty}^{+}$and $\Omega B \mathcal{M}$ when $\mathcal{M}$ is homotopy-commutative. Here the notation $X^{+}$denotes the Quillen plus construction with respect to the maximal perfect subgroup of $\pi_{1}(X),{ }^{2} \Omega$ is the based loop space functor, and $B$ is the classifying space functor. However, some details are missing from [MS75] to extract a complete proof of this fact. One needs to know first that the action of $\mathcal{M}$ on $\mathcal{M}_{\infty}$ induced by left-multiplication is by abelian homology equivalences when $\mathcal{M}$ is homotopy-commutative. A detailed proof of this has been written in [RW13]. Second, one needs to know that McDuff and Segal's groupcompletion theorem (Proposition 2 of [MS75]) is also true when "homology equivalence" and "homology fibration" are replaced by "abelian homology equivalence" and

${ }^{2}$ In this case, it follows from the fact that $\pi_{1}\left(\mathcal{M}_{\infty}^{+}\right) \cong \pi_{1}(\Omega B \mathcal{M})$ is abelian that the maximal perfect subgroup of $\pi_{1}\left(\mathcal{M}_{\infty}\right)$ is its commutator subgroup. This can also be proved directly; see Proposition 3.1 of $[\mathbf{R W} \mathbf{1 3}]$. 
"abelian homology fibration." This twisted version of the group-completion theorem is proved as Theorem 4.1 of [MP14]. ${ }^{3}$

Putting this together, we indeed have a weak equivalence $\mathcal{M}_{\infty}^{+} \simeq \Omega B \mathcal{M}$ (cf. Corollary 1.2 of [RW13] and Corollary 4.2 of [MP14]). Since loop spaces are simple we deduce:

Proposition 2.4. For a homotopy-commutative monoid $\mathcal{M}$ the space $\mathcal{M}_{\infty}^{+}$is simple.

See [RW13] and $\S 4$ of [MP14] for a more detailed discussion of this result.

\section{Models for scanning and stabilization}

In this section we define concrete models for all our configuration and section spaces and define the stabilization and scanning maps in these models so that they commute on the nose. We also give the more geometric description of the covering space $\Gamma^{+}(M) \rightarrow \Gamma(M)$ promised in the introduction.

Fix a connected, smooth, $m$-dimensional manifold $M$. As mentioned in the introduction, the unordered configuration space $C_{k}(M)$ of $k$ points in $M$ is defined to be the quotient of $M^{k} \backslash \Delta_{f}$ by the action of the symmetric group $\Sigma_{k}$, where $\Delta_{f}$ is the fat diagonal $\left\{\left(p_{1}, \ldots, p_{k}\right) \mid p_{i}=p_{j}\right.$ for some $\left.i \neq j\right\}$. The space $\Gamma(M)$ is defined to be the space of compactly supported sections of $\dot{T} M$, the fiberwise one-point compactification of the tangent bundle of $M$, given the subspace topology from the compact-open topology on $\operatorname{Map}(M, \dot{T} M)$. In $\S 3.1$ we define the degree of such a section; we denote by $\Gamma_{k}(M)$ the subspace (in fact, path component) of $\Gamma(M)$ of sections of degree $k \in \mathbb{Z}$.

We then define the models for the scanning and stabilization maps to be used in $\S \S 4-6$. For this some auxiliary data is needed. Given a Riemannian metric $g$ on $M$ and a smooth function $\rho: M \rightarrow(0,1)$ that is a strict lower bound for the injectivity radius of $(M, g)$, we define a scanning map

$$
s=s(g, \rho): C_{k}(M) \longrightarrow \Gamma_{k}(M) .
$$

To define the stabilization maps we first define alternative models for the configuration and section spaces, depending on the following auxiliary data. First, we choose an embedding $e: M \hookrightarrow(-1,1)^{d} \times \mathbb{R}$ for some $d$ such that

$$
e(M) \cap \pi_{d+1}^{-1}((-3, \infty))=(-1,1)^{m-1} \times(-3, \infty),
$$

where $\pi_{d+1}$ is the projection onto the $(d+1)$ st coordinate. Such an embedding exists if and only if $M$ is non-compact. This, in particular, chooses an "end" of $M$-namely, a proper homotopy class of properly embedded rays in $M$. We will also refer to $(-1,1)^{m-1} \times(-3, \infty)$ or its pre-image under $e$ as the chosen end of $M$. Second, we choose a Riemannian metric $g$ and function $\rho$ as above such that the ball around $p$ in $M$ of radius $\rho(p)$ does not extend too far in the $(d+1)$ st coordinate (see $\S 3.3$ for

\footnotetext{
${ }^{3}$ More generally, Theorem 4.1 of [MP14] proves a version of the group completion theorem where "homology equivalence" and "homology fibration" are replaced by "C્-homology equivalence" and " $\mathfrak{C}$-homology fibration" for any class $\mathfrak{C}$ of twisted coefficient systems which is closed under taking pullbacks. In contrast, the result of $[\mathbf{R W} 13]$ that $\mathcal{M}$ acts on $\mathcal{M}_{\infty}$ by abelian homology equivalences is sharp: it is in general not true that $\mathcal{M}$ acts on $\mathcal{M}_{\infty}$ by twisted homology equivalences, i.e., acyclic maps.
} 
the precise condition). The Riemannian metric is not required to be the one inherited from $(-1,1)^{d} \times \mathbb{R}$ via the embedding $e$; the purpose of the embedding is simply to (a) choose an end of the manifold and (b) provide convenient coordinates for that end.

Given such data $(e, g, \rho)$, we define in $\S 3.2$ spaces $C_{k}(M, e)$ and $\Gamma_{k}(M, e)$ and construct homotopy equivalences $C_{k}(M) \simeq C_{k}(M, e)$ and $\Gamma_{k}(M) \simeq \Gamma_{k}(M, e)$. In $\S 3.3$ we construct stabilization maps $t: C_{k}(M, e) \rightarrow C_{k+1}(M, e)$ and $T: \Gamma_{k}(M, e) \rightarrow$ $\Gamma_{k+1}(M, e)$ and also a scanning map $s: C_{k}(M, e) \rightarrow \Gamma_{k}(M, e)$. The square

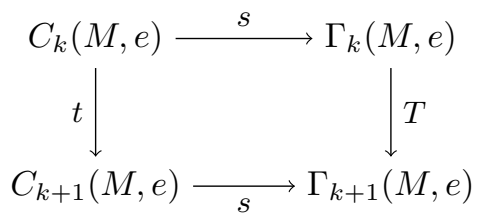

commutes on the nose (see $\S 3.5$ ), and the square

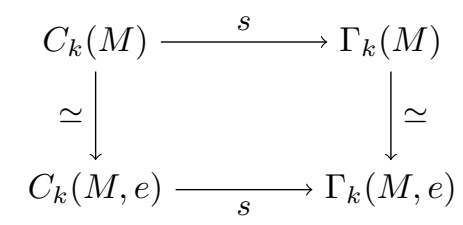

(where the top map is (3.1)) commutes up to a homotopy described in $\S 3.3$. In $\S 3.4$ we explain the modifications needed to define the stabilization map for oriented configuration spaces.

In $\S 3.6$ we geometrically construct a homomorphism $\pi_{1}\left(\Gamma_{k}(M, e)\right) \rightarrow \mathbb{Z} / 2$. This determines double covers (up to isomorphism) of all the spaces in the square (3.4) above, and the induced double cover of $C_{k}(M)$ is the oriented configuration space. Hence this definition of $\Gamma_{k}^{+}(M)$ agrees with the introduction, where it was characterized as the unique double cover that pulls back along the scanning map to the oriented configuration space.

Remark 3.1. In $\S \S 4$ and 5 we use the models $C_{k}(M, e), \Gamma_{k}(M, e)$, etc., whereas in $\S 6$ we use $C_{k}(M), \Gamma_{k}(M)$ and the model (3.1) of the scanning map. If we choose a particular double cover $\Gamma_{k}^{+}(M, e)$ in the isomorphism class that we have defined and pull this back in the square (3.4) to get particular double covers of the other spaces, then we get two double covers of $C_{k}(M)$ and the homotopy filling (3.4) determines an isomorphism between them. Temporarily denoting these two double covers by $C_{k}^{\top}(M)$ and $C_{k}^{\perp}(M)$, the homotopy in (3.4) also determines a homotopy filling the square:

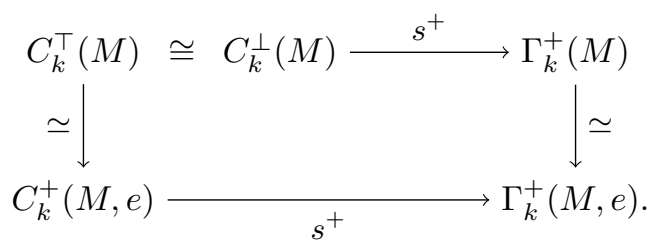

Hence for the purposes of $\S 6$ (showing that the lift $s^{+}$of the scanning map to the 
double covers is an isomorphism on homology in a range), the two models for $s^{+}$are equivalent.

\subsection{Degree of a section.}

We begin by defining the degree of a compactly supported section of $\pi: \dot{T} M \rightarrow M$. Let $\mathcal{O}_{M}$ denote the orientation bundle of $M$-in other words, the bundle of abelian groups whose fiber over $m \in M$ is $H_{d}(M, M \backslash\{m\} ; \mathbb{Z})$. Similarly, let $\mathcal{O}_{\dot{T} M}$ denote the orientation bundle of the manifold $\dot{T} M$-i.e, the bundle whose fiber over $\widetilde{m} \in \dot{T} M$ is $H_{2 d}(\dot{T} M, \dot{T} M \backslash\{\widetilde{m}\} ; \mathbb{Z})$.

Let $\sigma$ be a section of $\pi: \dot{T} M \rightarrow M$. We then have two bundles over $M$-namely, $\mathcal{O}_{M}$ and $\sigma^{*} \mathcal{O}_{\dot{T} M}$-which we claim are canonically isomorphic: Over $m \in M$ their fibers are $H_{d}(M, M \backslash\{m\} ; \mathbb{Z})$ and $H_{2 d}(\dot{T} M, \dot{T} M \backslash\{\sigma(m)\} ; \mathbb{Z})$ respectively. These are of course abstractly isomorphic, but, moreover, excision and the suspension isomorphism induce canonical isomorphisms for each $m$ which assemble to form a bundle isomorphism $\mathcal{O}_{M} \cong \sigma^{*} \mathcal{O}_{\dot{T} M}$.

Now we have a class $u \in H_{c}^{d}\left(\dot{T} M ; \mathcal{O}_{\dot{T} M}\right)$ that is the Poincaré dual to the zero section $M \subset \dot{T} M$. Pulling this back along the induced map $\sigma^{*}$ on compactly supported cohomology gives a class $\sigma^{*} u \in H_{c}^{d}\left(M ; \sigma^{*} \mathcal{O}_{\dot{T} M}\right) \cong H_{c}^{d}\left(M ; \mathcal{O}_{M}\right)$. Applying the Poincaré duality isomorphism, we therefore have a class in $H_{0}(M ; \mathbb{Z})=\mathbb{Z}$. The degree of $\sigma$ is defined to be this integer. Given two sections $\sigma, \tau \in \Gamma(M)$ in the same path component, the maps $\sigma^{*}$ and $\tau^{*}$ on compactly supported cohomology are equal, so $\operatorname{deg}(\sigma)=\operatorname{deg}(\tau)$. So this defines a map $\pi_{0}(\Gamma(M)) \rightarrow \mathbb{Z}$.

Denote the subspace of $\Gamma(M)$ of degree- $k$ sections by $\Gamma_{k}(M)$; by the previous paragraph this is a union of path components. Fix an open ball $B \subseteq M$ and note that the space of sections of $\pi$ with compact support in $B$ is homotopy equivalent to $\Omega^{d} S^{d}$. So clearly each $\Gamma_{k}(M)$ is non-empty since one can construct sections of any degree supported in $B$. Moreover, one can see that each $\Gamma_{k}(M)$ is path-connected as follows. First, as pointed out in [Lur09, Remark 3.8.8], each compactly supported section of $\pi$ is homotopic (through such sections) to one whose support is contained in $B .{ }^{4}$ Given two compactly-supported sections $\sigma$ and $\tau$ of degree $k$, one can homotope them to sections $\sigma^{\prime}$ and $\tau^{\prime}$ with compact support in $B$, which will again have degree $k$. The fact that the space $\Omega^{d} S^{d}$ has $\pi_{0}$ equal to $\mathbb{Z}$ (identified by the degree) means that one can homotope $\sigma^{\prime}$ to $\tau^{\prime}$ through sections supported in $B$.

Note that one could also define the degree of $\sigma \in \Gamma(M)$ using the fact mentioned in the previous paragraph: first, homotope $\sigma$ to a section $\sigma^{\prime}$ whose support is contained in $B$, so we can think of $\sigma^{\prime}$ as an element of $\Omega^{d} S^{d}$, and then define $\operatorname{deg}(\sigma):=\operatorname{deg}\left(\sigma^{\prime}\right)$. A priori this is not necessarily well defined, but in fact it is since it agrees with the definition given above.

\footnotetext{
${ }^{4}$ The argument is as follows: Let $M_{d-1} \subseteq M$ be the $(d-1)$-skeleton of a triangulation of $M$ (which exists since $M$ is smooth). The bundle $\left.\pi\right|_{M_{d-1}}$ has fibers whose connectivity is at least the dimension of its base, so by obstruction theory all sections of $\left.\pi\right|_{M_{d-1}}$ are homotopic (through sections). Therefore, any section of $\pi$ may be homotoped to be supported in $M \backslash M_{d-1}$, which is a union of disjoint open balls. Since the section was compactly supported to begin with, this new section will have support contained in a finite union of these open balls, and therefore contained in some (maybe larger) open ball in $M$. This can then be homotoped to be supported inside the given ball $B$.
} 


\subsection{Configuration and section spaces.}

We now define alternative models for configuration and section spaces, that will allow us to define stabilization and scanning maps that commute on the nose. Recall that we defined $C_{k}(M)$ as the quotient space $\left(M^{k} \backslash \Delta_{f}\right) / \Sigma_{k}$, where $\Delta_{f}$ is the fat diagonal in $M^{k}$, and $\Gamma(M)$ as the space of compactly supported sections of $\dot{T} M \rightarrow M$. We write $\Gamma_{k}(M)$ for the path component of $\Gamma(M)$ consisting of sections of degree $k$ and $C(M)$ for the disjoint union of $C_{k}(M)$ over all integers $k \geqslant 0$.

Assume that we have an embedding $e: M \hookrightarrow(-1,1)^{d} \times \mathbb{R}$ satisfying (3.2). Write $\hat{M}=e(M)$, and for $t \in[-3, \infty)$ define $\hat{M}_{t}:=e(M) \cap \pi_{d+1}^{-1}((-\infty, t))$. Then we define

$$
\begin{aligned}
& C_{k}(M, e):=\left\{(c, t) \in C_{k}(\hat{M}) \times[0, \infty) \mid c \subseteq \hat{M}_{t}\right\} \\
& \Gamma_{k}(M, e):=\left\{(\sigma, t) \in \Gamma_{k}(\hat{M}) \times[0, \infty) \mid \operatorname{supp}(\sigma) \subseteq \hat{M}_{t}\right\},
\end{aligned}
$$

which we give the subspace topology inherited from the product topology on $C_{k}(\hat{M}) \times$ $[0, \infty)$ and $\Gamma(\hat{M}) \times[0, \infty)$, respectively.

To justify this definition we will construct homotopy equivalences

$$
\bar{\imath}_{C}: C_{k}(M) \longleftrightarrow C_{k}(M, e): \bar{\jmath}_{C} \quad \text { and } \quad \bar{\imath}_{\Gamma}: \Gamma_{k}(M) \longleftrightarrow \Gamma_{k}(M, e): \bar{\jmath}_{\Gamma}
$$

for any $\operatorname{such}(M, e)$. First choose a continuous map $\phi:[0, \infty) \rightarrow \operatorname{Emb}((-3, \infty),(-3, \infty))$ such that $\phi_{t}((-3, \infty))=(-3, t)$ and $\phi_{t}$ is the identity near -3 . This induces a continuous map $\bar{\phi}:[0, \infty) \rightarrow \operatorname{Emb}(\hat{M}, \hat{M})$ via

$$
\bar{\phi}_{t}(p)= \begin{cases}p & p \in \hat{M} \backslash U \\ \left(q, \phi_{t}(s)\right) & p=(q, s) \in U,\end{cases}
$$

where $U=(-1,1)^{m-1} \times(-3, \infty)$. Also choose a trivialization $\psi: \dot{T} U \rightarrow U \times S^{d}$ over $U$. Then define $\bar{\imath}_{C}(c)=\left(\bar{\phi}_{0}(e(c)), 0\right)$ and $\bar{\jmath}_{C}(c, t)=e^{-1}\left(\bar{\phi}_{t}^{-1}(c)\right)$. The composition $\bar{\jmath}_{C} \circ$ $\bar{\imath}_{C}$ is the identity and a homotopy id $\Rightarrow \bar{\imath}_{C} \circ \bar{\jmath}_{C}$ is given by $(s, c, t) \mapsto\left(\bar{\phi}_{s t}\left(\bar{\phi}_{t}^{-1}(c)\right), s t\right)$. So $\bar{\imath}_{C}$ and $\bar{\jmath}_{C}$ are homotopy inverse, as required. We may similarly define $\bar{\imath}_{\Gamma}(\sigma)=$ $\left(\sigma^{\prime}, 0\right)$ where

$$
\sigma^{\prime}= \begin{cases}\sigma & \text { on } \hat{M} \backslash U \\ \psi^{-1} \circ\left(\bar{\phi}_{0} \times \text { id }\right) \circ \psi \circ \sigma \circ \bar{\phi}_{0}^{-1} & \text { on }(-1,1)^{m-1} \times(-3,0) \\ \infty & \text { on }(-1,1)^{m-1} \times[0, \infty)\end{cases}
$$

and

$$
\bar{\jmath}_{\Gamma}(\sigma, t)= \begin{cases}\sigma & \text { on } \hat{M} \backslash U \\ \psi^{-1} \circ\left(\bar{\phi}_{t}^{-1} \times \mathrm{id}\right) \circ \psi \circ \sigma \circ \bar{\phi}_{t} & \text { on } U,\end{cases}
$$

where we are implicitly identifying $M$ with $\hat{M}=e(M)$. The composition $\bar{\jmath}_{\Gamma} \circ \bar{\imath}_{\Gamma}$ is the identity, and a homotopy id $\Rightarrow \bar{\imath}_{\Gamma} \circ \bar{\jmath}_{\Gamma}$ is given by

$$
(s, \sigma, t) \mapsto \begin{cases}\sigma & \text { on } \hat{M} \backslash U \\ \psi^{-1} \circ\left(\bar{\phi}_{s t} \times \mathrm{id}\right) \circ\left(\bar{\phi}_{t}^{-1} \times \mathrm{id}\right) \circ \psi \circ \sigma \circ \bar{\phi}_{t} \circ \bar{\phi}_{s t}^{-1} & \text { on }(-1,1)^{m-1} \times(-3, s t) \\ \infty & \text { on }(-1,1)^{m-1} \times[s t, \infty) .\end{cases}
$$

Hence $\bar{\imath}_{\Gamma}$ and $\bar{\jmath}_{\Gamma}$ are also homotopy inverse, as required. 


\subsection{Stabilization and scanning maps.}

We now define the stabilization maps for configuration and section spaces, and two models for the scanning map, each depending on some auxiliary data. The stabilization map for configuration spaces depends on an embedding $e$ of $M$ into $(-1,1)^{d} \times \mathbb{R}$ that is prescribed at one end (so, in particular, $M$ must be open). The first model for the scanning map depends on a Riemannian metric $g$ and a function $\rho: M \rightarrow(0,1)$ and is defined for both open and closed manifolds. Finally, the stabilization map for section spaces and the second model for the scanning map depend on choices of $e, g$, and $\rho$ (so $M$ must be open).

We first define the stabilization map for configuration spaces, assuming that we have an embedding $e: M \hookrightarrow(-1,1)^{d} \times \mathbb{R}$ satisfying (3.2). As before, we write $\hat{M}=$ $e(M)$.

Definition 3.2. Define the stabilization map $t: C_{k}(M, e) \rightarrow C_{k+1}(M, e)$ by

$$
(c, t) \mapsto\left(c \cup\left\{\left(\mathbf{0}, t+\frac{1}{2}\right)\right\}, t+1\right),
$$

where $\mathbf{0}$ denotes $(0, \ldots, 0) \in(-1,1)^{d}$. Using the identifications of $\S 3.2$, this also determines a "stabilization map" $\bar{\jmath}_{C} \circ t \circ \bar{\imath}_{C}: C_{k}(M) \rightarrow C_{k+1}(M)$ for any smooth, connected, non-compact manifold $M$, depending on the choice of embedding $e$.

We next define the scanning map for any Riemannian manifold with a choice of lower bound for its injectivity radius. Let $g$ be a Riemannian metric on $M$, and let $\rho: M \rightarrow(0,1)$ be a strict lower bound for its injectivity radius. Let d denote the metric (in the sense of a distance function) induced by $g$, and for $p \in M$ and $r>0$ define $B_{r}(p)$ to be $\{q \in M \mid \mathrm{d}(p, q)<r\}$. If $r \in(0, \rho(p)]$ the exponential map for $M$ restricts to a homeomorphism from the open $r$-ball in $T_{p} M$ to $B_{r}(p)$. Composing the inverse of this with the dilation $v \mapsto\left(\frac{1}{r-|v|}-\frac{1}{r}\right) \cdot v$ defines a homeomorphism

$$
\mathcal{E}_{p, r}: B_{r}(p) \rightarrow T_{p} M
$$

We also define a function $\epsilon: C_{k}(M) \times M \rightarrow[0,1]$ by

$$
\epsilon(c, p):=\sup \left\{r \in[0,1] \mid r \leqslant \rho(p) \text { and }\left|B_{r}(p) \cap c\right| \leqslant 1\right\} .
$$

Note that the subset $\left\{r \in[0,1] \mid r \leqslant \rho(p)\right.$ and $\left.\left|B_{r}(p) \cap c\right| \leqslant 1\right\}$ of $[0,1]$ is closed and hence compact, so $\epsilon$ is a continuous function and $\left|B_{\epsilon(c, p)}(p) \cap c\right| \leqslant 1$ for all $(c, p)$. Also note that $\epsilon(c, p)>0$ for all $(c, p)$.

Definition 3.3. Define the scanning map $s: C_{k}(M) \rightarrow \Gamma_{k}(M)$ by $s(c)=\sigma$, where

$$
\sigma(p)= \begin{cases}\mathcal{E}_{p, \epsilon(c, p)}(q) \in T_{p} M & \text { if } B_{\epsilon(c, p)}(p) \cap c=\{q\} \\ \infty & \text { if } B_{\epsilon(c, p)}(p) \cap c=\varnothing .\end{cases}
$$

We now define a second model for the scanning map, of the form $s: C_{k}(M, e) \rightarrow$ $\Gamma_{k}(M, e)$, depending on some auxiliary data $(e, g, \rho)$. Choose an embedding $e: M \hookrightarrow$ $(-1,1)^{m-1} \times \mathbb{R}$ satisfying (3.2), a Riemannian metric $g$ on $M$, and a smooth function $\rho: M \rightarrow(0,1)$ that is a strict lower bound for the injectivity radius of $(M, g)$. The extra property that we require this to satisfy is that the radius- $\rho$ balls in $M$ do not extend too far in the $(d+1)$ st coordinate direction, which is formulated precisely as follows. As previously, we write $U=(-1,1)^{m-1} \times(-3, \infty)$ for the chosen end of the 
manifold $\hat{M}=e(M)$ and implicitly identify $M$ with $\hat{M}$. For points $p \in U$ we require that

$$
B_{\rho(p)}(p) \subseteq(-1,1)^{d} \times\left(x-\frac{1}{8}, x+\frac{1}{8}\right),
$$

where $x=\pi_{d+1}(p)$, and for points $p \in \hat{M} \backslash U$ we require that

$$
B_{\rho(p)}(p) \subseteq(-1,1)^{d} \times\left(-\infty,-3+\frac{1}{8}\right) .
$$

Definition 3.3 applied to $\hat{M}$ and the data $(g, \rho)$ (transferred to $\hat{M}$ via $e$ ) gives a scanning map which we denote by $\hat{s}: C_{k}(\hat{M}) \rightarrow \Gamma_{k}(\hat{M})$.

Definition 3.4. Define the model $s: C_{k}(M, e) \rightarrow \Gamma_{k}(M, e)$ for the scanning map by

$$
(c, t) \mapsto\left(\hat{s}(c), t+\frac{1}{4}\right) .
$$

To describe a homotopy filling the square (3.4) we first define a "shifting" operation on compactly supported sections of $\hat{M}$ that roughly conjugates a section by the selfembedding $\bar{\phi}_{t}: \hat{M} \hookrightarrow \hat{M}$. For $t \in[0, \infty)$ and $\sigma \in \Gamma(\hat{M})$ define

$$
\Phi_{t}(\sigma)= \begin{cases}\sigma & \text { on } \hat{M} \backslash U \\ \psi^{-1} \circ\left(\bar{\phi}_{t} \times \text { id }\right) \circ \psi \circ \sigma \circ \bar{\phi}_{t}^{-1} & \text { on }(-1,1)^{m-1} \times(-3, t) \\ \infty & \text { on }(-1,1)^{m-1} \times[t, \infty) .\end{cases}
$$

For example, in $\S 3.2$ we have $\bar{\imath}_{C}(\sigma)=\left(\Phi_{0}(\sigma), 0\right)$. Now, the two ways around the square (3.4) take a configuration $c \in C_{k}(M)$ to

$$
\left(\hat{s}\left(\bar{\phi}_{0}(c)\right), \frac{1}{4}\right) \quad \text { and } \quad\left(\Phi_{0}(\hat{s}(c)), 0\right),
$$

respectively. To fill in a homotopy between these maps one can define it to take a configuration $c \in C_{k}(M)$ to

$$
\left(\Phi_{\tanh (t)}\left(\hat{s}\left(\bar{\phi}_{\tanh (1-t)}(c)\right)\right), \frac{t}{4}\right)
$$

for $t \in(0,1)$. More informally, the two routes around (3.4) either scan the configuration and then compress the resulting section so that its support lies in $\hat{M}_{0}$, or, conversely compress the configuration so that it lies in $\hat{M}_{0}$ and then scan it. The homotopy interpolates between these two by compressing the configuration into $\hat{M}_{t}$ for $t \in(0, \infty)$, scanning, and then further compressing the resulting section into $\hat{M}_{0}$.

Definition 3.5. Suppose we have an embedding $e: M \hookrightarrow(-1,1)^{d} \times \mathbb{R}$ satisfying (3.2), as well as a Riemannian metric $g$ on $M$ and a strict lower bound $\rho: M \rightarrow(0,1)$ for its injectivity radius satisfying (3.8) and (3.9). Define the stabilization map $T: \Gamma_{k}(M, e) \rightarrow$ $\Gamma_{k+1}(M, e)$ by $T(\sigma, t)=\left(\sigma^{\prime}, t+1\right)$ where

$$
\sigma^{\prime}(p)= \begin{cases}\sigma(p) & \text { if } p \in \hat{M}_{t} \\ \mathcal{E}_{p, \rho(p)}(q) & \text { if } q \in B_{\rho(p)}(p) \\ \infty & \text { otherwise, }\end{cases}
$$

where $q=\left(\mathbf{0}, t+\frac{1}{4}\right)$. Using the identifications of $\S 3.2$, this also determines a "stabilization map" $\bar{\jmath}_{\Gamma} \circ T \circ \bar{\imath}_{\Gamma}: \Gamma_{k}(M) \rightarrow \Gamma_{k+1}(M)$ for any smooth, connected, non-compact manifold $M$, depending on the choice of embedding $e$. 
Reverse stabilization. In the definition above, the restriction of $\sigma^{\prime}$ to the strip

$$
\hat{M}_{t+1} \backslash \hat{M}_{t}=(-1,1)^{m-1} \times[t, t+1)
$$

has degree 1. We could insert a reflection into the definition of $\sigma^{\prime}$ so that it instead has degree -1 , and thereby define a "reverse stabilization map" $U: \Gamma_{k}(M, e) \rightarrow$ $\Gamma_{k-1}(M, e)$. It is not hard to show that $U$ is a homotopy inverse for $T$, so, in particular, the homotopy type of $\Gamma_{k}(M, e)$ is independent of $k$.

\subsection{Oriented configuration spaces.}

Recall that the oriented configuration spaces are defined by $C_{k}^{+}(M)=\left(M^{k} \backslash\right.$ $\left.\Delta_{f}\right) / A_{k}$ and double-cover the unordered configuration spaces $C_{k}(M)$. The homotopy equivalence $C_{k}(M) \simeq C_{k}(M, e)$ constructed in $\S 3.2$ clearly lifts to a homotopy equivalence $C_{k}^{+}(M) \simeq C_{k}^{+}(M, e)$, where $C_{k}^{+}(M, e)$ is the double cover of $C_{k}(M, e)$ defined by

$$
C_{k}^{+}(M, e):=\left\{(c, t) \in C_{k}^{+}(\hat{M}) \times[0, \infty) \mid c \subseteq \hat{M}_{t}\right\} .
$$

There are exactly two lifts of the stabilization map $t: C_{k}(M, e) \rightarrow C_{k+1}(M, e)$ to a map $C_{k}^{+}(M, e) \rightarrow C_{k+1}^{+}(M, e)$; they differ by the deck transformation that reverses the orientation of a configuration, in other words, the transformation that permutes an ordered configuration representing an element of $C_{k+1}^{+}(M, e)$ by an odd permutation. We choose $t^{+}: C_{k}^{+}(M, e) \rightarrow C_{k+1}^{+}(M, e)$ to be the one defined by

$$
\left(\left[p_{1}, \ldots, p_{k}\right], t\right) \mapsto\left(\left[p_{1}, \ldots, p_{n},\left(\mathbf{0}, t+\frac{1}{2}\right)\right], t+1\right),
$$

where [-] denotes a finite-ordered set modulo even permutations.

\subsection{Scanning commutes with stabilization.}

The stabilization and scanning maps were defined in $\S 3.3$ in order to commute on the nose, as the following lemma proves.

Lemma 3.6. The following square commutes:

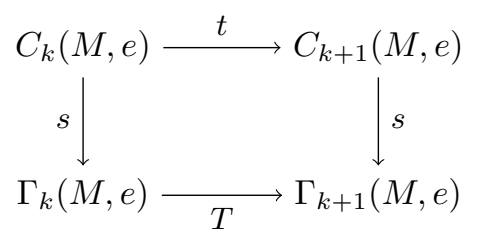

An illustration of the two ways around this square is given in Figure 3.1.

Proof. Let $(c, t) \in C_{k}(M, e)$. Then

$$
\begin{aligned}
t(c, t) & =\left(c_{1}, t+1\right) & s\left(c_{1}, t+1\right) & =\left(\sigma_{1}, t+\frac{5}{4}\right) \\
s(c, t) & =\left(\sigma_{0}, t+\frac{1}{4}\right) & T\left(\sigma_{0}, t+\frac{1}{4}\right) & =\left(\sigma_{2}, t+\frac{5}{4}\right),
\end{aligned}
$$

where $c_{1}=c \cup\{q\}$ for $q=\left(\mathbf{0}, t+\frac{1}{2}\right)$. We need to show that $\sigma_{1}=\sigma_{2}$. 
First, consider $p \in \hat{M} \backslash \hat{M}_{t+\frac{1}{4}}$. In this case, we have $B_{\rho(p)}(p) \subseteq \hat{M} \backslash \hat{M}_{t}$ by (3.8), so $B_{\rho(p)}(p) \cap c_{1}$ can only be either $\{q\}$ or $\varnothing$. Hence $\epsilon\left(c_{1}, p\right)=\rho(p)$ and

$$
\sigma_{1}(p)=\hat{s}\left(c_{1}\right)(p)= \begin{cases}\mathcal{E}_{p, \rho(p)}(q) & \text { if } q \in B_{\rho(p)}(p) \\ \infty & \text { otherwise. }\end{cases}
$$

This is also the value of $\sigma_{2}(p)$, by definition of $T$, so $\sigma_{1}$ and $\sigma_{2}$ agree on this subspace.

For $p \in \hat{M}_{t+\frac{1}{4}}$ the value of $\sigma_{2}(p)=\sigma_{0}(p)$ is given by (3.7) with $M \mapsto \hat{M}$, and $\sigma_{1}(p)$ is given by the same formula with $c$ replaced by $c_{1}$. To show that these agree it suffices to show that $\epsilon(c, p)=\epsilon\left(c_{1}, p\right)$ and $B_{\epsilon(c, p)}(p) \cap c=B_{\epsilon(c, p)}(p) \cap c_{1}$. Note that the value of $\epsilon(c, p)$ depends only on $p$ and the subconfiguration $B_{\rho(p)}(p) \cap c$ of $c$. The symmetric difference of $c$ and $c_{1}$ is $\{q\}$, but by (3.8) we have $q \notin B_{\rho(p)}(p)$. Hence we have

$$
B_{\rho(p)}(p) \cap c=B_{\rho(p)}(p) \cap c_{1},
$$

so $\epsilon(c, p)=\epsilon\left(c_{1}, p\right)$. Since $\epsilon(c, p) \leqslant \rho(p)$, we also have $q \notin B_{\epsilon(c, p)}(p)$, and so

$$
B_{\epsilon(c, p)}(p) \cap c=B_{\epsilon(c, p)}(p) \cap c_{1} .
$$

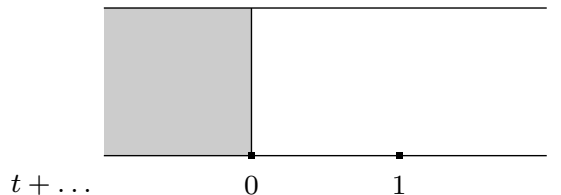

\}

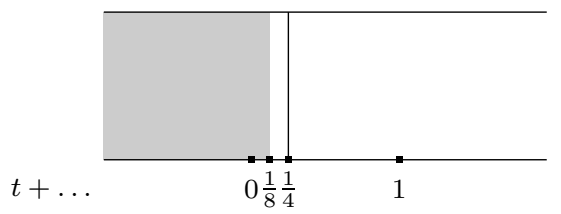

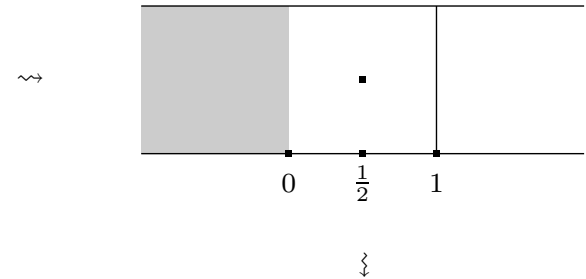

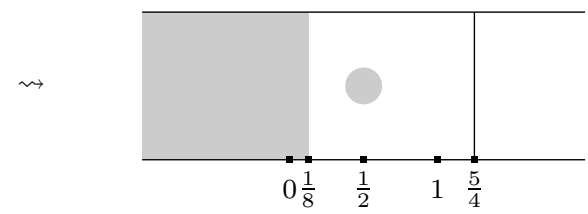

Figure 3.1: The effect of the maps of the square (3.10) on a point in $C_{k}(M, e)$. The pictures show the segment $(-1,1)^{d} \times(t-1, t+2)$ of $\hat{M}$. The configuration (resp. support of the section) is contained in the shaded region, except in the top-right, where there is one extra configuration point, as indicated.

\subsection{A double cover of the section space.}

We now geometrically construct a homomorphism $\pi_{1}\left(\Gamma_{k}(M, e)\right) \rightarrow \mathbb{Z} / 2$. This defines double covers of all the spaces in the square (3.4), and it will be constructed so that the double cover of $C_{k}(M)$ that it determines is the oriented configuration space $C_{k}^{+}(M)$.

To do this we construct a map $e_{\Gamma}: \Gamma_{k}(M, e) \rightarrow \Gamma_{k}\left((-1,1)^{d} \times \mathbb{R}\right)$ such that the 
square

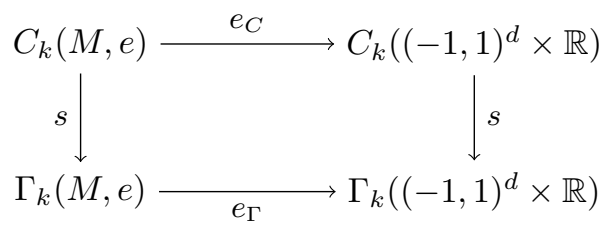

commutes up to homotopy, where the left-hand $s$ is as in Definition 3.4, the righthand $s$ is as in Definition 3.3, and $e_{C}(c, t)=c$. This will achieve the aim of this subsection by the following. The bottom right-hand space is homotopy equivalent to $\Omega_{k}^{d+1} S^{d+1}$, so, since $d \geqslant 2$, it has fundamental group $\mathbb{Z} / 2$. The map on $\pi_{1}$ induced by $e_{\Gamma}$ is then the desired homomorphism $\pi_{1}\left(\Gamma_{k}(M, e)\right) \rightarrow \mathbb{Z} / 2$, defining a double cover $\Gamma_{k}^{+}(M, e)$ of $\Gamma_{k}(M, e)$. We want the pullback of this double cover along $s \circ$ $\bar{\imath}_{C}: C_{k}(M) \rightarrow C_{k}(M, e) \rightarrow \Gamma_{k}(M, e)$ to be the oriented configuration space $C_{k}^{+}(M)$. Note that on fundamental groups the right-hand vertical map is the sign homomorphism $\Sigma_{k} \rightarrow \mathbb{Z} / 2,{ }^{5}$ so this is isomorphic to the pullback along $e_{C} \circ \bar{\imath}_{C}$ of the double cover of $C_{k}\left((-1,1)^{d} \times \mathbb{R}\right)$ corresponding to the alternating group $A_{k} \leqslant \Sigma_{k}$. Hence it is indeed the oriented configuration space.

It thus remains to construct $e_{\Gamma}$ and see that (3.11) commutes up to homotopy. We will construct $e_{\Gamma}$ in detail but leave the homotopy-commutativity of the square as an exercise for the reader, since the proofs of our main results do not depend on the equivalence of the two definitions of $\Gamma_{k}^{+}(M)$.

Definition 3.7. For brevity, write $E=(-1,1)^{d} \times \mathbb{R}$, so we have an embedding $e: M \hookrightarrow$ $E$, and let $\pi: \nu \rightarrow M$ denote the normal bundle of this embedding. Choose a tubular neighborhood for $e$-in other words, an embedding $\bar{e}: \nu \hookrightarrow E$ so that $\bar{e} \circ z=e$, where $z$ is the zero section of the normal bundle also satisfying the following additional condition. The restrictions of tangent bundles $\left.T \nu\right|_{z(M)}$ and $\left.T E\right|_{e(M)}$ are both canonically isomorphic to $\nu \oplus T M$, and the bundle map $T \bar{e}:\left.\left.T \nu\right|_{z(M)} \rightarrow T E\right|_{e(M)}$ is of the form $\phi \oplus$ id under these identifications for some bundle automorphism $\phi$ of $\nu$; we require that $\phi=$ id.

Let $(\sigma, t) \in \Gamma_{k}(M, e)$, so in particular $\sigma \in \Gamma(\hat{M})$. We then define

$$
e_{\Gamma}(\sigma, t):=\sigma^{\prime} \in \Gamma(E)
$$

as follows. For $p \in E$ not in the tubular neighborhood $\bar{e}(\nu)$, let $\sigma^{\prime}(p)=\infty$. Now let $p=\bar{e}(x)$ for some $x \in \nu$. We want to define $\sigma^{\prime}(p)$ in $\dot{T}_{p} E$, which is identified via $\bar{e}$ with $\dot{T}_{x} \nu$. Note that the bundle $T \nu$ decomposes as $\pi^{*} \nu \oplus \pi^{*} T M$, so what we need is an element of the one-point compactification of $\nu_{\pi(x)} \oplus T_{\pi(x)} M$. But we have the element

$$
(-x, \sigma(\pi(x))) \in \nu_{\pi(x)} \oplus \dot{T}_{\pi(x)} M,
$$

which lives in a one-point partial compactification of $\nu_{\pi(x)} \oplus T_{\pi(x)} M$ and therefore

${ }^{5}$ Assuming that $d \geqslant 2$ and $k \geqslant 2$. The former ensures that $\pi_{1}\left(C_{k}\left(\mathbb{R}^{d+1}\right)\right) \cong \Sigma_{k}$ and that $\pi_{1}\left(\Omega_{\bullet}^{d+1} S^{d+1}\right) \cong \mathbb{Z} / 2$. The latter plus McDuff's theorem (Theorem 1.3) implies that the map is surjective on $H_{1}$, and therefore also on $\pi_{1}$ since $\pi_{1}$ of the codomain is abelian. The only surjective map $\Sigma_{k} \rightarrow \mathbb{Z} / 2$ is the sign homomorphism. 
also gives an element in its one-point compactification. This defines a section $\sigma^{\prime} \in$ $\Gamma(E)$, and one can check that $\operatorname{deg}\left(\sigma^{\prime}\right)=\operatorname{deg}(\sigma)$, so in fact $\sigma^{\prime} \in \Gamma_{k}(E)$.

This is the formal definition of $\sigma^{\prime}$. The idea is to extend the section $\sigma$ to the tubular neighborhood $\bar{e}(\nu)$ so that its value goes to infinity as you go to infinity in the fiber direction of the normal bundle $\nu$, so that we can then extend it by infinity outside the tubular neighborhood. To achieve this we define $\sigma^{\prime}(x)$, for a point $x$ in the normal bundle over $\pi(x) \in M$, to be the value of $\sigma$ on $\pi(x)$ plus another term which goes to infinity as $x$ goes to infinity. Using the decomposition of the tangent bundle of the normal bundle explained above, we can take this extra term to be $-x$. See Figure 3.2.

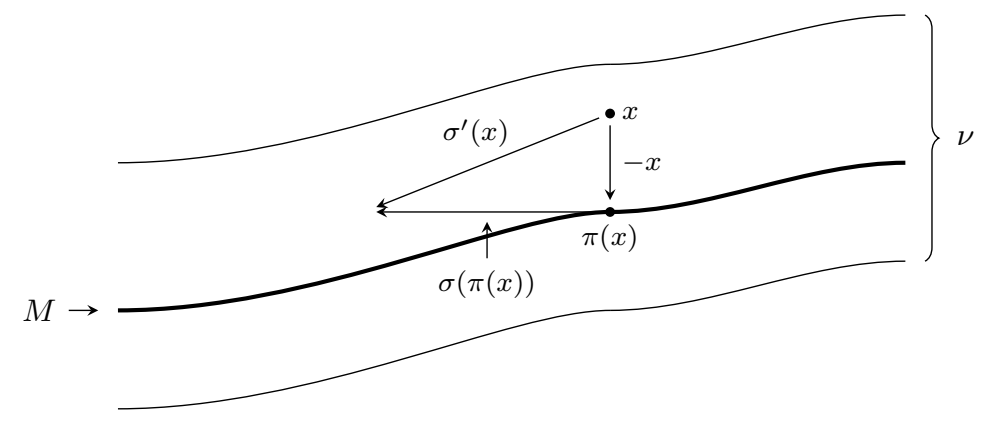

Figure 3.2: The definition of $\sigma^{\prime}$ on a point $x$ in the normal bundle $\nu$. This defines $\sigma^{\prime}$ on the tubular neighborhood $\bar{e}(\nu)$, and outside this tubular neighborhood $\sigma^{\prime}$ is defined to be $\infty$.

\section{Euclidean spaces}

Let $(M, e)$ be as in $\S 3$. The sequences of stabilization maps for configuration and section spaces for $k \geqslant 0$ determine mapping telescopes $\operatorname{Tel}\left(C_{k}(M, e)\right)$ and $\operatorname{Tel}\left(\Gamma_{k}(M, e)\right)$, and since the scanning maps commute with stabilization maps there is an induced map of mapping telescopes $s: \operatorname{Tel}\left(C_{k}(M, e)\right) \rightarrow \operatorname{Tel}\left(\Gamma_{k}(M, e)\right)$. The precise statement of Theorem $\mathrm{B}$ is that this map is acyclic.

Write $C(M, e)=\bigsqcup_{k \in \mathbb{N}} C_{k}(M, e)$ and $\Gamma(M, e)=\bigsqcup_{k \in \mathbb{Z}} \Gamma_{k}(M, e)$. The stabilization maps $t$ and $T$ are endomorphisms of $C(M, e)$ and $\Gamma(M, e)$, respectively, and we can also form mapping telescopes $\operatorname{Tel}(C(M, e))$ and $\operatorname{Tel}(\Gamma(M, e))$ with respect to these endomorphisms. The scanning map $s: C(M, e) \rightarrow \Gamma(M, e)$ commutes with these endomorphisms and therefore induces a map of mapping telescopes $s_{\mathrm{Tel}}: \operatorname{Tel}(C(M, e)) \rightarrow$ $\operatorname{Tel}(\Gamma(M, e))$. There is a commutative square

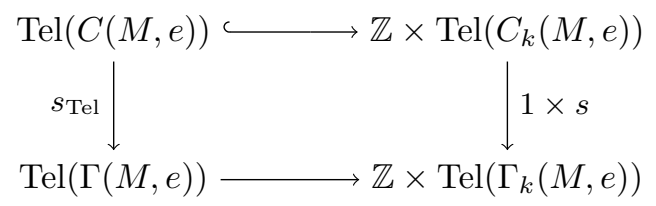

in which the horizontal maps are homotopy equivalences, so it is equivalent to prove that the map $s_{\mathrm{Tel}}: \operatorname{Tel}(C(M, e)) \rightarrow \operatorname{Tel}(\Gamma(M, e))$ is acyclic. 
In this section we prove this in the special case $(M, e)=\left(E^{m}, \iota\right)$ for $d \geqslant m \geqslant 2$, where $E^{m}=(-1,1)^{m-1} \times \mathbb{R}$ and $\iota$ is the inclusion of $E^{m}$ into $(-1,1)^{d} \times \mathbb{R}$. The main technical input for this is the following.

Lemma 4.1. The spaces $\Gamma\left(E^{m}, \iota\right)$ and $\operatorname{Tel}\left(C\left(E^{m}, \iota\right)\right)^{+}$are simple, where $(-)^{+}$denotes Quillen plus-construction with respect to the maximal perfect subgroup.

Before proving this we show how it implies Theorem B in the special case.

Proof of Theorem B when $(M, e)=\left(E^{m}, \iota\right)$. Recall from $\S 3.3$ that the stabilization maps $T: \Gamma_{k}(M, e) \rightarrow \Gamma_{k+1}(M, e)$ are homotopy equivalences for any $(M, e)$, so we have a homotopy equivalence $\operatorname{Tel}(\Gamma(M, e)) \simeq \Gamma(M, e)$. Also, by McDuff's theorem [McD75, Theorem 1.2] (Theorem 1.2 in the introduction) the map

$$
s_{\mathrm{Tel}}: \operatorname{Tel}(C(M, e)) \longrightarrow \operatorname{Tel}(\Gamma(M, e))
$$

is a homology equivalence (for untwisted integral coefficients) for any $(M, e)$.

Lemma 4.1 tells us that $\operatorname{Tel}\left(\Gamma\left(E^{m}, \iota\right)\right)$ and $\operatorname{Tel}\left(C\left(E^{m}, \iota\right)\right)^{+}$are simple spaces, so applying Quillen's plus-construction to the map $(4.2)$ for $(M, e)=\left(E^{m}, \iota\right)$ results in a homology equivalence between simple spaces. This is a weak equivalence by the homology Whitehead theorem, so the original map (4.2) is acyclic.

Proof of Lemma 4.1. We will write $J=(-1,1)$. Recall that

$$
\begin{aligned}
& C_{k}\left(E^{m}, \iota\right)=\left\{(c, t) \in C_{k}\left(J^{m-1} \times \mathbb{R}\right) \times[0, \infty) \mid c \subseteq J^{m-1} \times(-\infty, t)\right\} \\
& \Gamma_{k}\left(E^{m}, \iota\right)=\left\{(\sigma, t) \in \Gamma_{k}\left(J^{m-1} \times \mathbb{R}\right) \times[0, \infty) \mid \operatorname{supp}(\sigma) \subseteq J^{m-1} \times(-\infty, t)\right\} .
\end{aligned}
$$

We define subspaces

$$
\begin{aligned}
\mathcal{M}_{k} & :=\left\{(c, t) \in C_{k}\left(J^{m-1} \times \mathbb{R}\right) \times[0, \infty) \mid c \subseteq J^{m-1} \times(0, t)\right\} \\
\mathcal{N}_{k} & :=\left\{(\sigma, t) \in \Gamma_{k}\left(J^{m-1} \times \mathbb{R}\right) \times[0, \infty) \mid \operatorname{supp}(\sigma) \subseteq J^{m-1} \times(0, t)\right\},
\end{aligned}
$$

and let $\mathcal{M}=\bigsqcup_{k \in \mathbb{N}} \mathcal{M}_{k}$ and $\mathcal{N}=\bigsqcup_{k \in \mathbb{Z}} \mathcal{N}_{k}$.

For $t \in \mathbb{R}$ define $\lambda_{t}$ to be the automorphism $(x, u) \mapsto(x, u+t)$ of $J^{m-1} \times \mathbb{R}$. Also choose a trivialization of the bundle $\dot{T}\left(J^{m-1} \times \mathbb{R}\right)$, i.e., a homeomorphism $\psi: \dot{T}\left(J^{m-1} \times\right.$ $\mathbb{R}) \rightarrow J^{m-1} \times \mathbb{R} \times S^{m}$ over $J^{m-1} \times \mathbb{R}$. Then we can define monoid structures on $\mathcal{M}$ and $\mathcal{N}$ by

$$
\begin{aligned}
\left(c_{1}, t_{1}\right) \cdot\left(c_{2}, t_{2}\right) & =\left(c_{1} \cup \lambda_{t_{1}}\left(c_{2}\right), t_{1}+t_{2}\right) \\
\left(\sigma_{1}, t_{1}\right) \cdot\left(\sigma_{2}, t_{2}\right) & =\left(\sigma_{3}, t_{1}+t_{2}\right),
\end{aligned}
$$

where $\sigma_{3}$ is defined by

$$
\sigma_{3}= \begin{cases}\sigma_{1} & \text { on } J^{m-1} \times\left(0, t_{1}\right) \\ \psi^{-1} \circ\left(\lambda_{t_{1}} \times \mathrm{id}\right) \circ \psi \circ \sigma_{2} \circ \lambda_{-t_{1}} & \text { on } J^{m-1} \times\left(t_{1}, t_{1}+t_{2}\right) \\ \infty & \text { otherwise. }\end{cases}
$$

Note that $\pi_{0}(\mathcal{M})=\mathbb{N}$ and $\pi_{0}(\mathcal{N})=\mathbb{Z}$, so $\mathcal{N}$ is grouplike.

The inclusions $\mathcal{M}_{k} \hookrightarrow C_{k}\left(E^{m}, \iota\right)$ and $\mathcal{N}_{k} \hookrightarrow \Gamma_{k}\left(E^{m}, \iota\right)$ are homotopy equivalences; for example, one can define homotopy inverses as follows. Choose an order-preserving homeomorphism $\phi:(-1,0) \rightarrow(-\infty, 0)$, and for $t \in(0, \infty)$ define a homeomorphism 
$\bar{\phi}_{t}: J^{m-1} \times(0, t) \rightarrow J^{m-1} \times(-\infty, t)$ by $\bar{\phi}_{t}(x, u)=\left(x, \phi\left(\frac{u}{t}-1\right)+t\right)$. We can then define homotopy inverses by $(c, t) \mapsto\left(\bar{\phi}_{t}^{-1}(c), t\right)$ and $(\sigma, t) \mapsto\left(\sigma^{\prime}, t\right)$, where

$$
\sigma^{\prime}= \begin{cases}\psi^{-1} \circ\left(\bar{\phi}_{t}^{-1} \times \mathrm{id}\right) \circ \psi \circ \sigma \circ \bar{\phi}_{t} & \text { on } J^{m-1} \times(0, t) \\ \infty & \text { otherwise. }\end{cases}
$$

Hence we have a homotopy equivalence $\mathcal{N} \simeq \Gamma\left(E^{m}, \iota\right)$. Since $\mathcal{N}$ is a grouplike monoid - in particular, a grouplike $\mathrm{H}$-space - it is simple, and therefore so is $\Gamma\left(E^{m}, \iota\right)$.

The monoid $\mathcal{M}$ is not grouplike, but it is homotopy-commutative since $m \geqslant 2$ (this can be shown analogously to the proof that higher homotopy groups are abelian). Let $m_{1} \in \mathcal{M}_{1}$ be the element $\left(\left\{\left(\mathbf{0}, \frac{1}{2}\right)\right\}, 1\right)$. Note that the restriction of the stabilization map $t: C_{k}\left(E^{m}, \iota\right) \rightarrow C_{k+1}\left(E^{m}, \iota\right)$ to $\mathcal{M}_{k}$ is precisely right-multiplication by $m_{1}$. Hence the square

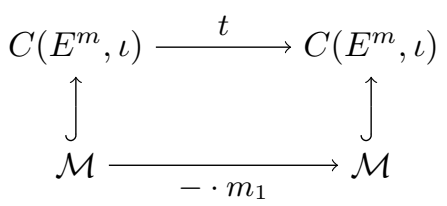

commutes (and the vertical maps are homotopy equivalences), so we have a homotopy equivalence $\operatorname{Tel}(\mathcal{M}) \hookrightarrow \operatorname{Tel}\left(C\left(E^{m}, \iota\right)\right)$. By Proposition 2.4, the Quillen plusconstruction $\operatorname{Tel}(\mathcal{M})^{+}$is a simple space. Hence $\operatorname{Tel}\left(C\left(E^{m}, \iota\right)\right)^{+}$is also simple.

\section{Manifolds admitting boundary}

We begin with some notation for relative configuration and section spaces. Let $N$ be a smooth manifold with boundary $\partial N$, and let $A \subseteq N$ be a subset.

Definition 5.1. Define the relative configuration space $C(N, A)$ to be the quotient of $C(N)$ in which we identify two configurations if they agree on $N \backslash A$. Define the relative section space $\Gamma(N, A)$ to be the space of sections $s$ of $\left.\dot{T} N\right|_{N \backslash A} \rightarrow N \backslash A$ such that $\{x \in N \backslash A \mid s(x) \neq \infty\}$ is contained in a compact subset of $N$ and such that $s(x)=\infty$ for all $x \in \partial N$. Note that if $N \backslash A$ is a codimension 0 submanifold of $N$ that is closed as a subspace, then $\Gamma(N, A)$ is all compactly supported sections of $\dot{T}(N \backslash A)$ that restrict to $\infty$ on $\partial N \backslash A \subseteq \partial(N \backslash A)$ but not necessarily on $\partial(N \backslash$ $A) \backslash \partial N$. When $A=\varnothing$ we write $C(N, \varnothing)=C(N)$ and $\Gamma(N, \varnothing)=\Gamma(N)$. This agrees with the previous definition of $\Gamma(N)$ when $N$ has empty boundary. There are natural maps $\pi: C(N) \rightarrow C(N, A)$ and $r: \Gamma(N) \rightarrow \Gamma(N, A)$, defined by $\pi(c)=[c]$ and $r(\sigma)=$ $\left.\sigma\right|_{N \backslash A}$.

Now let $M$ be any connected, smooth, non-compact manifold of dimension $m \geqslant 2$, and pick an embedding $e: M \hookrightarrow(-1,1)^{d} \times \mathbb{R}$ satisfying (3.2). As usual, we write $\hat{M}=e(M)$.

Definition 5.2. Define $\bar{M}=\hat{M} \cup\left([-1,1]^{m-1} \times(-3, \infty]\right)$ and $\bar{M}_{t}=\bar{M} \cap \pi_{d+1}^{-1}((-\infty, t))$. 
Note that $\operatorname{int}\left(\bar{M}_{t}\right)=\hat{M}_{t}$. Define $\Gamma(\bar{M}, e)$ analogously to $\Gamma(M, e)$; in other words,

$$
\Gamma(\bar{M}, e)=\left\{(\sigma, t) \in \Gamma(\bar{M}) \times[0, \infty) \mid \operatorname{supp}(\sigma) \subseteq \bar{M}_{t}\right\} .
$$

Also, let $B_{1}=(-1,1)^{m-1} \times[-1, \infty)$ and $B_{2}=[-1,1]^{m-1} \times(-2, \infty]$, and define

$$
\begin{aligned}
& \mathbf{C}(M, e)=C\left(\hat{M}, B_{1}\right) \\
& \boldsymbol{\Gamma}(M, e)=\Gamma\left(\hat{M}, \stackrel{\circ}{B}_{2}\right) \quad \text { where } \stackrel{\circ}{B}_{2}=\operatorname{int}\left(B_{2}\right) \\
& \boldsymbol{\Gamma}(\bar{M}, e)=\Gamma\left(\bar{M}, B_{2}\right) .
\end{aligned}
$$

There are maps $C(M, e) \rightarrow C(M), \Gamma(M, e) \rightarrow \Gamma(M)$, and $\Gamma(\bar{M}, e) \rightarrow \Gamma(\bar{M})$ which forget the extra parameter $t$. Composing these with the maps $\pi$ and $r$ from Definition 5.1, we obtain maps $\pi: C(M, e) \rightarrow \mathbf{C}(M, e), r: \Gamma(M, e) \rightarrow \boldsymbol{\Gamma}(M, e)$, and $\bar{r}: \Gamma(\bar{M}, e) \rightarrow$ $\boldsymbol{\Gamma}(\bar{M}, e)$.

There is a map $i: \Gamma(M, e) \rightarrow \Gamma(\bar{M}, e)$ given by extending a compactly supported section of $\hat{M}$ by $\infty$ on $\partial \bar{M}$. Choosing an isotopy from the identity to an embedding $\bar{M} \hookrightarrow \bar{M}$ taking $\partial \bar{M}$ into the interior determines a homotopy inverse for $i$. One can similarly define a homotopy equivalence $\mathbf{i}: \boldsymbol{\Gamma}(M, e) \rightarrow \boldsymbol{\Gamma}(\bar{M}, e)$, and these commute: $\bar{r} \circ i=\mathbf{i} \circ r$.

Now also choose a Riemannian metric $g$ on $M$ and a strict lower bound $\rho: M \rightarrow$ $(0,1)$ for its injectivity radius satisfying conditions (3.8) and (3.9). In $\S 3.3$ we defined stabilization and scanning maps depending on this auxiliary data. The stabilization map $T: \Gamma(M, e) \rightarrow \Gamma(M, e)$ can be extended to a map $T: \Gamma(\bar{M}, e) \rightarrow \Gamma(\bar{M}, e)$ using exactly the same formula as in Definition 3.5, so that $T \circ i=i \circ T$.

Lemma 5.3. There is a well-defined map $\mathbf{s}: \mathbf{C}(M, e) \rightarrow \boldsymbol{\Gamma}(M, e)$ making the left-hand square below commute on the nose:

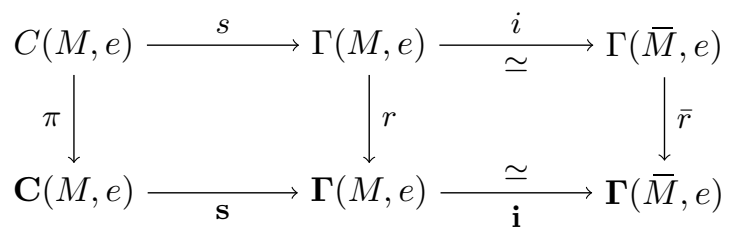

Proof. Let $\left(c_{i}, t_{i}\right) \in C(M, e)$ for $i=1,2$, so we have two sections $\sigma_{i}=r \circ s\left(c_{i}, t_{i}\right)$ of $\dot{T}\left(M \backslash B_{2}\right)$. Let $p \in M \backslash B_{2}$. We need to show that $\left[c_{1}\right]=\left[c_{2}\right]$ implies that $\sigma_{1}(p)=$ $\sigma_{2}(p)$. Note that, by definition of the scanning map $s, \sigma_{i}(p)$ depends only on $c_{i} \cap$ $B_{\rho(p)}(p)$, where $\rho: M \rightarrow(0,1)$ was part of the auxiliary data used to define scanning. But conditions (3.8) and (3.9) imply that $B_{\rho(p)}(p)$ is contained in $M \backslash B_{1}$ and, by assumption, $c_{1}$ and $c_{2}$ agree on $M \backslash B_{1}$.

Let $\operatorname{Tel}(C(M, e))$ be the mapping telescope of the infinite sequence of stabilization maps $t: C(M, e) \rightarrow C(M, e) \rightarrow C(M, e) \rightarrow \cdots$. Note that $\pi \circ t=\pi$, so there is a well-defined map $\pi_{\mathrm{Tel}}: \operatorname{Tel}(C(M, e)) \rightarrow \mathbf{C}(M, e)$. We can similarly use the stabilization maps for $\Gamma(M, e)$ and $\Gamma(\bar{M}, e)$ to obtain maps $r_{\mathrm{Tel}}: \operatorname{Tel}(\Gamma(M, e)) \rightarrow \boldsymbol{\Gamma}(M, e)$ and $\bar{r}_{\mathrm{Tel}}: \operatorname{Tel}(\Gamma(\bar{M}, e)) \rightarrow \boldsymbol{\Gamma}(\bar{M}, e)$. The scanning map commutes with the stabilization maps (§3.5), as does the map $i$, so there are induced maps $s_{\mathrm{Tel}}$ and $i_{\mathrm{Tel}}$ and a 
commutative square

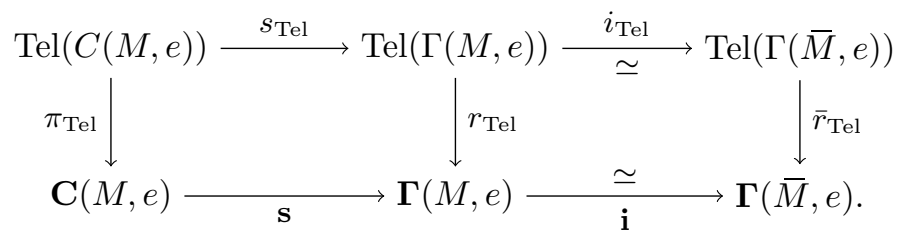

There are four ingredients for the proof in this section of Theorem B: One of these is the special case of Theorem B proved in $\S 4$ where $(M, e)=\left(E^{m}, \iota\right)$. The other three are the following lemma:

Lemma 5.4. In the square (5.2),

(a) $\pi_{\mathrm{Tel}}$ is an abelian homology fibration,

(b) $\bar{r}_{\text {Tel }}$ is a quasifibration,

(c) $\mathbf{s}$ is a weak equivalence.

Before proving these we will show how they imply Theorem B.

Proof of Theorem B. Recall that $E^{m}=(-1,1)^{m-1} \times \mathbb{R}$ and $\iota$ is the inclusion of this into $(-1,1)^{d} \times \mathbb{R}$. In $\S 4$ we proved Theorem B for $(M, e)=\left(E^{m}, \iota\right)$-namely, that the scanning map $s_{\mathrm{Tel}}: \operatorname{Tel}\left(C\left(E^{m}, \iota\right)\right) \rightarrow \operatorname{Tel}\left(\Gamma\left(E^{m}, \iota\right)\right)$ is acyclic.

Let $\varnothing \in \mathbf{C}(M, e)$ denote the empty relative configuration, and let $\varnothing \in \boldsymbol{\Gamma}(M, e)$ and $\varnothing \in \boldsymbol{\Gamma}(\bar{M}, e)$ denote the infinity sections of $\dot{T}\left(\hat{M} \backslash \stackrel{\circ}{B}_{2}\right)$ and of $\dot{T}\left(\bar{M} \backslash B_{2}\right)$, respectively. The point-set fiber over $\varnothing \in \mathbf{C}(M, e)$ in (5.1) is

$$
\begin{aligned}
\pi^{-1}(\varnothing) & =\left\{(c, t) \in C(\hat{M}) \times[0, \infty) \mid c \subseteq B_{1} \cap \hat{M}_{t}\right\} \quad \subset C(M, e) \\
& \cong\left\{(c, t) \in C\left(\hat{E}^{m}\right) \times[0, \infty) \mid c \subseteq B_{1} \cap \hat{E}_{t}^{m}\right\} \quad \subset C\left(E^{m}, \iota\right),
\end{aligned}
$$

and similarly the point-set fiber $r^{-1}(\varnothing)$ may be thought of as a subspace of $\Gamma\left(E^{m}, \iota\right)$. Moreover, the inclusions $\pi^{-1}(\varnothing) \hookrightarrow C\left(E^{m}, \iota\right)$ and $r^{-1}(\varnothing) \hookrightarrow \Gamma\left(E^{m}, \iota\right)$ are homotopy equivalences: in each case, one can construct a homotopy inverse using an isotopy $h_{t}$ of embeddings $\hat{E}^{m} \hookrightarrow \hat{E}^{m}$ from the identity to an embedding $h_{1}$ with image equal to $(-1,1)^{m-1} \times(-1, \infty)$.

The stabilization map $t: C\left(E^{m}, \iota\right) \rightarrow C\left(E^{m}, \iota\right)$ restricts to an endomorphism of $\pi^{-1}(\varnothing)$, so we can form the mapping telescope $\operatorname{Tel}\left(\pi^{-1}(\varnothing)\right)$ with respect to this endomorphism, and the inclusion $\operatorname{Tel}\left(\pi^{-1}(\varnothing)\right) \hookrightarrow \operatorname{Tel}\left(C\left(E^{m}, \iota\right)\right)$ is a homotopy equivalence. ${ }^{6}$ Similarly, using the stabilization map $T$ for section spaces, we have a mapping telescope $\operatorname{Tel}\left(r^{-1}(\varnothing)\right)$ and an inclusion $\operatorname{Tel}\left(r^{-1}(\varnothing)\right) \hookrightarrow \operatorname{Tel}\left(\Gamma\left(E^{m}, \iota\right)\right)$ which is a homotopy equivalence. The following square commutes on the nose:

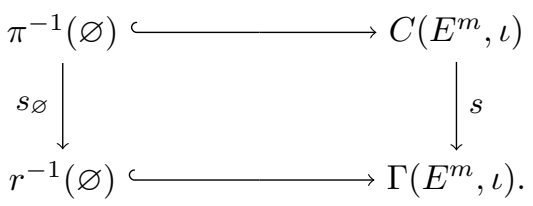

${ }^{6}$ Since the homotopy inverse to the inclusion $\pi^{-1}(\varnothing) \hookrightarrow C\left(E^{m}, \iota\right)$ can be constructed to commute with the stabilization map $t$ by ensuring that the isotopy $h_{t}$ is the identity on $(-1,1)^{m-1} \times[0, \infty)$ at all times $t$. 
A little care is needed here: Implicitly, we have chosen a Riemannian metric $g_{M}$ and a function $\rho_{M}: M \rightarrow(0,1)$ to define the scanning map $C(M, e) \rightarrow \Gamma(M, e)$, which restricts to the map $s_{\varnothing}$ in this square. We then choose the corresponding data $\left(g_{E}, \rho_{E}\right)$, which determines $C\left(E^{m}, \iota\right) \rightarrow \Gamma\left(E^{m}, \iota\right)$, to agree with $\left(g_{M}, \rho_{M}\right)$ on $\stackrel{\circ}{B}_{2}$, which ensures that the square commutes. Hence the square after taking mapping telescopes,

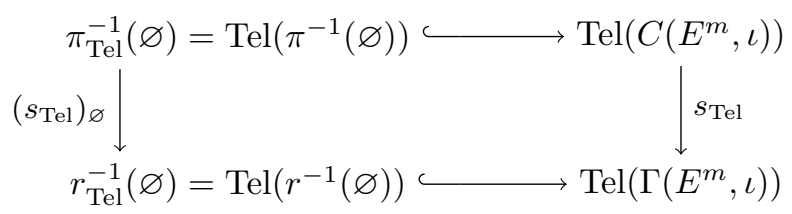

also commutes. The map $s_{\mathrm{Tel}}$ is acyclic by $\S 4$, and the horizontal maps are homotopy equivalences, so $\left(s_{\mathrm{Tel}}\right)_{\varnothing}$ is also acyclic.

Now consider the squares

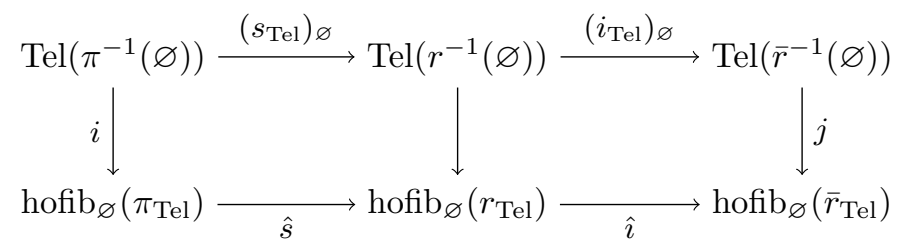

which compare the set-theoretic and homotopy fibers of the squares (5.2). By Lemma 5.4 $i$ is an abelian homology equivalence and $j$ is a weak equivalence, and by above $\left(s_{\text {Tel }}\right)_{\varnothing}$ is acyclic. The map $\left(i_{\mathrm{Tel}}\right)_{\varnothing}$ may be compared to the map $i_{\mathrm{Tel}}$ for $(M, e)=\left(E^{m}, \iota\right)$ similarly to (5.3), so it is a homotopy equivalence. Hence the bottom horizontal map $\hat{\imath} \circ \hat{s}$ is also an abelian homology equivalence. Moreover, recall from Lemma 4.1 that $\Gamma\left(E^{m}, \iota\right)$ is a simple space - in particular, its fundamental group is abelian. Therefore, the same is true for $r^{-1}(\varnothing)$, therefore also for each path component of $\operatorname{Tel}\left(r^{-1}(\varnothing)\right)$, and therefore also for each path-component of hofib $\varnothing\left(\bar{r}_{T e l}\right)$. So all local coefficient systems on hofib $\varnothing\left(\bar{r}_{\text {Tel }}\right)$ are abelian, so the map $\hat{\imath} \circ \hat{s}$ is in fact acyclic.

Finally, consider the diagram obtained from the outer square of (5.2) by taking homotopy fibers horizontally and vertically. By Lemma $5.4 \mathrm{~s}$ is a weak equivalence, and therefore so is the map hofib $(\hat{\imath} \circ \hat{s}) \rightarrow \operatorname{hofib}\left(i_{\mathrm{Tel}} \circ s_{\mathrm{Tel}}\right)$ in this diagram. Since acyclicity of a map $f$ is equivalent to hofib $(f)$ having the integral (untwisted) homology of a point, the fact that $\hat{\imath} \circ \hat{s}$ is acyclic implies that $i_{\mathrm{Tel}} \circ s_{\mathrm{Tel}}$ is also acyclic. But $i_{\mathrm{Tel}}$ is a homotopy equivalence, so $s_{\mathrm{Tel}}$ is acyclic, as required.

It now remains to prove Lemma 5.4. We prove part (a) first, and we begin by describing the mapping telescope $\operatorname{Tel}(C(M, e))$ a little more.

Definition 5.5. Define $C_{\infty}(M, e)$ to be the quotient of

$$
\left\{(c, t, u) \in C(\hat{M}) \times[0, \infty)^{2} \mid c \subseteq \hat{M}_{t} \text { and }|c| \leqslant u \leqslant|c|+1\right\}
$$

by the equivalence relation $\sim$ that is the reflexive, symmetric closure of

$$
(c, t,|c|) \sim\left(c \cup\left\{p_{t}\right\}, t+1,|c|+1\right),
$$

where for $t \in[0, \infty), p_{t}:=\left(\mathbf{0}, t+\frac{1}{2}\right) \in \hat{M}$. This is homeomorphic to the mapping 
telescope of the sequence of stabilization maps $C_{0}(M, e) \rightarrow C_{1}(M, e) \rightarrow \cdots$, which was denoted in $\S 4$ by $\operatorname{Tel}\left(C_{k}(M, e)\right)$. There is a descending filtration $C_{\infty}(M, e) \supset$ $C_{\infty-1}(M, e) \supset \cdots \supset C_{\infty-k}(M, e) \supset \cdots$ defined by

$$
C_{\infty-k}(M, e)=\left\{[c, t, u] \in C_{\infty}(M, e)|| c \mid \geqslant k\right\} .
$$

There is a small ambiguity here: to make this unambiguous we specify that the element $[c, t,|c|]=\left[c \cup\left\{p_{t}\right\}, t+1,|c|+1\right]$ is included in $C_{\infty-k}(M, e)$ when $|c|=k-1$. This corresponds to the mapping telescope of the subsequence $C_{k}(M, e) \rightarrow C_{k+1}(M, e) \rightarrow$ $C_{k+2}(M, e) \rightarrow \cdots$.

The mapping telescope $\operatorname{Tel}(C(M, e))$ is homeomorphic to the disjoint union

$$
\bigsqcup_{k \in \mathbb{Z}} C_{\infty-\max (k, 0)}(M, e)
$$

and we will often talk of the element $[c, t, u]$ of $\operatorname{Tel}(C(M, e))$, eliding the $k$ that specifies the path component. Under this identification, on each path component $C_{\infty-k}(M, e)$ the map $\pi_{\text {Tel }}$ restricts to the map $C_{\infty-k}(M, e) \rightarrow \mathbf{C}(M, e)$ given by $[c, t, u] \mapsto[c]$.

Lemma 5.6. The map $\pi_{\mathrm{Tel}}$ is an abelian homology fibration.

Proof. Write $Y=\operatorname{Tel}(C(M, e))$ and $X=\mathbf{C}(M, e)$. Define

$$
X_{n}=\left\{[c] \in \mathbf{C}(M, e)|| c \backslash B_{1} \mid \leqslant n\right\} .
$$

This is an increasing filtration of $X$ by closed subsets, and each compact subset of $X$ is contained in some $X_{n}$. Also, note that both $X$ and $Y$ are Hausdorff: for $X=\mathbf{C}(M, e)=C\left(\hat{M}, B_{1}\right)$ this uses the fact that $B_{1}$ is closed in $\hat{M}$ and that $\hat{M}$ is $T_{3}$ (since it is Hausdorff and locally compact). So we just need to verify the conditions (i)-(iii) of Proposition 2.3 in this case.

Since $X_{0}$ is a point, $\pi_{\mathrm{Tel}}$ is vacuously an abelian homology fibration over it. For all $n \geqslant 1$ the pre-image $\pi_{\text {Tel }}^{-1}\left(X_{n} \backslash X_{n-1}\right)$ is the subspace of $Y$ of elements $[c, t, u]$ where the configuration $c$ has exactly $n$ points in $\hat{M} \backslash B_{1}$. In this subspace no points can pass between $B_{1}$ and $\hat{M} \backslash B_{1}$, so $\pi_{\text {Tel }}$ restricted to this subspace is a trivial fiber bundle over $X_{n} \backslash X_{n-1}$, and so, in particular, an abelian homology fibration over $X_{n} \backslash X_{n-1}$. This verifies condition (ii).

For condition (iii), define $U_{n}$ to be the open subset of $X_{n}$ consisting of those configurations $c$ with $\left|c \backslash B_{1}\right| \leqslant n$ and $\left|c \backslash \stackrel{\circ}{B}_{2}\right| \leqslant n-1$. Let $f_{t}:(-3,0) \rightarrow(-3,0)$ be the following function:

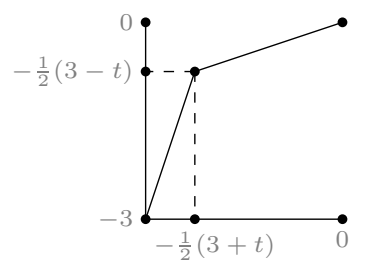

This induces an automorphism $g_{t}$ of $\hat{M}$ that is id $\times f_{t}$ on $(-1,1)^{m-1} \times(-3,0)$ and the identity elsewhere. We can then define the homotopies $h_{t}$ and $H_{t}$ needed for condition (iii) by simply applying $g_{t}$ to each point of the configuration or relative 
configuration. Properties (a) and (b) of these homotopies are immediate from their definition.

To check property (c) we will show that for $x \in U_{k}$ the map $H_{1}: \pi_{\text {Tel }}^{-1}(x) \rightarrow \pi_{\text {Tel }}^{-1}\left(h_{1}(x)\right)$ is an abelian homology equivalence. Recall from the proof of Theorem B above that there is an inclusion $\pi_{\mathrm{Tel}}^{-1}(\varnothing)=\operatorname{Tel}\left(\pi^{-1}(\varnothing)\right) \hookrightarrow \operatorname{Tel}\left(C\left(E^{m}, \iota\right)\right)$ which is a homotopy equivalence. Also, recall from the proof of Lemma 4.1 that there is a homotopycommutative monoid $\mathcal{M}$ with $\pi_{0}$ equal to $\mathbb{N}$ and an element $m_{1} \in \mathcal{M}$ (so that $\left[m_{1}\right] \in$ $\pi_{0}(\mathcal{M})$ is the generator) with the following property. If we write

$$
\operatorname{Tel}(\mathcal{M})=\operatorname{Tel}(\mathcal{M} \rightarrow \mathcal{M} \rightarrow \cdots),
$$

where each map $\mathcal{M} \rightarrow \mathcal{M}$ is right-multiplication by $m_{1}$, then there is an inclusion $\operatorname{Tel}(\mathcal{M}) \hookrightarrow \operatorname{Tel}\left(C\left(E^{m}, \iota\right)\right)$ which is a homotopy equivalence. Also note that the fibers of $\pi_{\mathrm{Tel}}$ are all canonically homeomorphic (this of course does not mean that it is locally trivial). Putting this together, we have the following (without the dotted arrows):

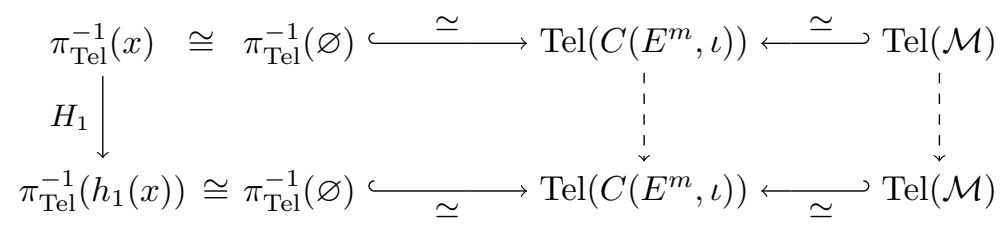

Now $x=[c] \in \mathbf{C}(M, e)$. Although $[c]$ is a relative configuration, it determines welldefined subconfigurations $c \backslash B_{1}$ and

$$
g_{1}(c) \backslash B_{1}=c \backslash g_{1}^{-1}\left(B_{1}\right)=c \backslash \operatorname{cl}\left(\stackrel{\circ}{B}_{2}\right),
$$

so we may define $\ell_{x}:=\left|c \backslash B_{1}\right|-\left|g_{1}(c) \backslash B_{1}\right| \in \mathbb{N}$. This is the number of points of the (relative) configuration $x$ that are pushed into $B_{1}$ during the homotopy $H_{t}$. The diagram (5.4) may be completed with dotted arrows so that it homotopy-commutes, and, moreover, the arrow $\operatorname{Tel}(\mathcal{M}) \rightarrow \operatorname{Tel}(\mathcal{M})$ is the map induced by left-multiplication $\left(m_{1}\right)^{\ell_{x}} \cdot-: \mathcal{M} \rightarrow \mathcal{M}$.

In $[\mathbf{R W 1 3}, \S 2]$ it is shown that for a homotopy-commutative monoid $\mathcal{M}$ and element $m_{1} \in \mathcal{M}$ so that $\left[m_{1}\right]$ generates $\pi_{0}(\mathcal{M})=\mathbb{N},{ }^{7}$ we have the following: for any element $m \in \mathcal{M}$ the endomorphism $\operatorname{Tel}(\mathcal{M}) \rightarrow \operatorname{Tel}(\mathcal{M})$ induced by left-multiplication $m \cdot-: \mathcal{M} \rightarrow \mathcal{M}$ is an abelian homology equivalence. Applying this to the above situation, we see that the right-hand vertical arrow of (5.4) is an abelian homology equivalence, and therefore so is $H_{1}: \pi_{\mathrm{Tel}}^{-1}(x) \rightarrow \pi_{\mathrm{Tel}}^{-1}\left(h_{1}(x)\right)$, proving property (c). This completes the verification of condition (iii).

To verify condition (i) we will show that $\pi_{\mathrm{Tel}}$ is locally stalk-like over each $X_{n}$. There is a basis for $X_{n}$ consisting of the following open sets. Choose $j \leqslant n$ small non-overlapping open discs in the manifold $\hat{M} \backslash B_{1}$ and a real number $\epsilon \in(-3,-1)$ such that no disc intersects $(-1,1)^{m-1} \times(\epsilon,-1)$. Then take the subspace $V$ of $X_{n}$ of relative configurations where each of the discs contains exactly one point, the strip $(-1,1)^{m-1} \times(\epsilon,-1)$ contains any configuration of at most $n-j$ points, and the rest of the manifold $\hat{M} \backslash B_{1}$ is empty. This subset $V$ is clearly contractible: one can

\footnotetext{
${ }^{7}$ The fact proved in $[\mathbf{R W 1 3}, \S 2]$ is more general, but slightly more complicated to state, than what
} we state here. In particular, it does not require that $\pi_{0}(\mathcal{M})=\mathbb{N}$. 
deformation retract it onto the configuration $v_{0} \in V$ in which the point in each disc is at its center and the strip $D \times(\epsilon,-1)$ is empty. The collection of all such $V$ forms a basis for $X_{n}$.

We now show that the inclusion $\pi_{\mathrm{Tel}}^{-1}\left(v_{0}\right) \hookrightarrow \pi_{\mathrm{Tel}}^{-1}(V)$ is a weak equivalence - in fact, a homotopy equivalence - we describe a homotopy inverse which lifts the deformation retraction of $V$ onto $\left\{v_{0}\right\} .{ }^{8}$ Observe that $\pi_{\text {Tel }}^{-1}\left(v_{0}\right)$ consists of elements $[c, t, u] \in$ $\operatorname{Tel}(C(M, e))$ such that

$$
c \backslash B_{1}=c \backslash\left((-1,1)^{m-1} \times[-1, \infty)\right)
$$

is precisely the $j$ centers of the discs. On the other hand, $\pi_{\text {Tel }}^{-1}(V)$ consists of elements $[c, t, u] \in \operatorname{Tel}(C(M, e))$ such that

$$
c \backslash\left((-1,1)^{m-1} \times(\epsilon, \infty)\right)
$$

consists of one point in each disc. There is a homotopy from the identity on $\pi_{\mathrm{Tel}}^{-1}(V)$ to a self-map with image inside $\pi_{\mathrm{Tel}}^{-1}\left(v_{0}\right)$ given by gradually pushing each point in a disc toward the center of that disc and gradually pushing the configuration in $(-1,1)^{m-1} \times(\epsilon, \infty)$ to the right until it lies in $(-1,1)^{m-1} \times(-1, \infty) \cdot{ }^{9}$

This proves that $\pi_{\mathrm{Tel}}$ is locally stalk-like over $X_{n}$, so we have verified condition (i) of Proposition 2.3. Hence $\pi_{\mathrm{Tel}}$ is an abelian homology fibration.

We now turn to part (b) of Lemma 5.4, for which we use the following general fact.

Lemma 5.7. Let $p: Y \rightarrow X$ be a Serre fibration with a chosen section $s_{0}: X \rightarrow Y$. Let $A \subseteq X$ be a relatively compact subset such that $(X, X \backslash A)$ is a relative $C W$ complex, and let $B \subseteq X \backslash A$ be any subset. Denote by $\Gamma_{B}(p)$ the space of all compactly supported sections of $p$ that agree with $s_{0}$ on $B$, and denote by $\Gamma_{B}(p, A)$ the space of all sections of $p^{-1}(X \backslash A) \rightarrow X \backslash A$ whose support is contained in a compact subset of $X$ and which agree with $s_{0}$ on $B$. Then the restriction map $\Gamma_{B}(p) \rightarrow \Gamma_{B}(p, A)$ is a Serre fibration.

Proof. Any lifting problem for the restriction map induces a lifting problem for $p$, as below:

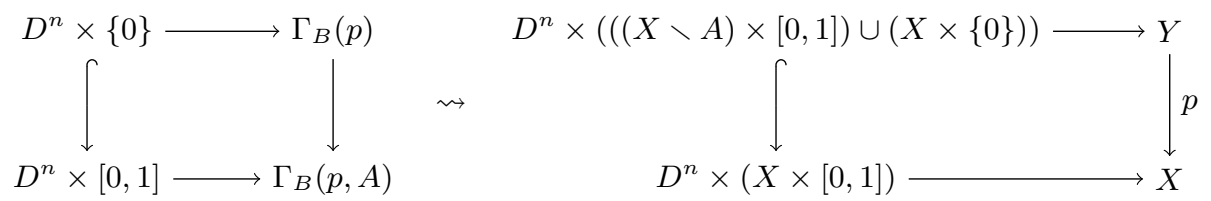

In the right-hand square the left vertical map is the inclusion of a relative CWcomplex and $p$ is a Serre fibration, so there is a diagonal map for this square. This gives a map $D^{n} \times[0,1] \rightarrow \Gamma_{B}^{n c}(p)$, where $\Gamma_{B}^{n c}(p)$ is the space of all (not necessarily

${ }^{8}$ This is not, however, a deformation retraction of $\pi_{\mathrm{Tel}}^{-1}(V)$ onto $\pi_{\mathrm{Tel}}^{-1}\left(v_{0}\right)$, since the subspace $\pi_{\mathrm{Tel}}^{-1}\left(v_{0}\right)$ is not fixed during the homotopy.

${ }^{9}$ Note that it is important that we do not need $\pi_{\mathrm{Tel}}^{-1}(v) \hookrightarrow \pi_{\mathrm{Tel}}^{-1}(V)$ to be a weak equivalence for all $v \in V$, since the previous argument does not work if the configuration $v$ has non-empty intersection with the strip $(-1,1)^{m-1} \times(\epsilon,-1)$. 
compact) sections of $p$ that agree with $s_{0}$ on $B$. If this map lands in the subspace $\Gamma_{B}(p)$, we will have found a diagonal for the left-hand square above.

In other words: the diagonal map $D^{n} \times(X \times[0,1]) \rightarrow Y$ restricted to a point in $D^{n} \times[0,1]$ is a section of $p$, and we need to show that it has compact support. But its restriction to $X \backslash A$ has support contained in a compact subset $K$ of $X$, so the support of the whole section must be contained in $K \cup \bar{A}$, which is compact since $A$ is relatively compact in $X$.

We also note that a mapping telescope of quasifibrations over a fixed base is again a quasifibration: more precisely, for a sequence of spaces $Y_{1} \rightarrow Y_{2} \rightarrow \cdots$ and quasifibrations $f_{i}: Y_{i} \rightarrow X$ commuting with the maps $Y_{i} \rightarrow Y_{i+1}$, the induced map $\operatorname{Tel}\left(f_{i}\right): \operatorname{Tel}\left(Y_{i}\right) \rightarrow X$ is a quasifibration. This is because (a) for any basepoint $x \in X$ we have hofib $x\left(\operatorname{Tel}\left(f_{i}\right)\right)=\operatorname{Tel}\left(\operatorname{hofib}_{x}\left(f_{i}\right)\right)$ and the subspace $\left(\operatorname{Tel}\left(f_{i}\right)\right)^{-1}(x)$ is the submapping telescope $\operatorname{Tel}\left(f_{i}^{-1}(x)\right)$, and (b) a telescope of weak equivalences is a weak equivalence.

The map $\bar{r}: \Gamma(\bar{M}, e) \rightarrow \boldsymbol{\Gamma}(\bar{M}, e)$ factors as

$$
\Gamma(\bar{M}, e) \longrightarrow \Gamma(\bar{M}) \longrightarrow \Gamma\left(\bar{M}, \stackrel{\circ}{B}_{2}\right) \longrightarrow \Gamma\left(\bar{M}, B_{2}\right)=\Gamma(\bar{M}, e) .
$$

Applying Lemma 5.7 to the fiber bundle $\dot{T} \bar{M} \rightarrow \bar{M}$ with the section at infinity, $A=$ $\dot{B}_{2}$ and $B=\partial \bar{M}$, we see that the middle map is a Serre fibration. The first map is a Hurewicz fibration: given a lifting problem $f: X \times\{0\} \rightarrow \Gamma(\bar{M}, e), g: X \times[0,1] \rightarrow$ $\Gamma(\bar{M})$, we can define a lift $h: X \times[0,1] \rightarrow \Gamma(\bar{M}, e)$ by

$$
h(x, t)=\left(g(x, t), m(g(x, t))+\operatorname{pr}_{2}(f(x, 0))-m\left(\operatorname{pr}_{1}(f(x, 0))\right)\right),
$$

where $m: \Gamma(\bar{M}) \rightarrow[0, \infty)$ takes a configuration to the maximum of the $(d+1)$ st coordinates of all its points and 0 . The third map is a homeomorphism, with inverse given by extending a section by $\infty$ on $\partial \bar{M} \cap B_{2}$.

Applying the above remark to the sequence of stabilization maps $\Gamma(\bar{M}, e) \rightarrow \Gamma(\bar{M}, e)$ $\rightarrow \cdots$, this implies that the map $\bar{r}_{\mathrm{Tel}}: \operatorname{Tel}(\Gamma(\bar{M}, e)) \rightarrow \boldsymbol{\Gamma}(\bar{M}, e)$ is a quasifibration. Thus, we have proved part (b) of Lemma 5.4.

As a final input for the proof of Theorem B we need to prove part (c) of Lemma 5.4namely, that the relative scanning map

$$
\text { s: } \mathbf{C}(M, e)=C\left(\hat{M}, B_{1}\right) \longrightarrow \Gamma\left(\hat{M}, \stackrel{\circ}{B}_{2}\right)=\mathbf{\Gamma}(M, e)
$$

is a weak equivalence.

Proposition 5.8. The relative scanning map $\mathbf{s}$ is a weak equivalence.

Proof. This essentially follows from Proposition 2 of [Böd87]. However, no explicit description of the relative scanning map is given there, so we will follow $\S 2.3$ of [Hes92], which contains an explicit description and generalizes Proposition 2 of [Böd87] to configurations with labels in a bundle over the manifold (although we will not need this).

Choose an increasing filtration of $\hat{M}$ by compact submanifolds-with-boundary $N_{n}$. Moreover, choose these so that

$$
N_{n} \cap \pi_{d+1}^{-1}((-3, \infty))=\left[\frac{1-n}{n}, \frac{n-1}{n}\right]^{m-1} \times(-3, n] .
$$

Also, define $P_{n}=B_{1} \cap N_{n}=\left[\frac{1-n}{n}, \frac{n-1}{n}\right]^{m-1} \times[-1, n]$. Write $s^{0}$ for the trivial $S^{0}$ bundle over $N_{n}$. In general, for a manifold $N$ with boundary $\partial N$ we write $N^{\circ}=$ 
$N \cup(\partial N \times[0,1))$. We then have

$$
\begin{aligned}
& C\left(N_{n}, P_{n}\right) \cong C\left(N_{n}, P_{n} ; s^{0}\right) \\
& \Gamma\left(N_{n}, P_{n}\right) \cong \Gamma\left(N_{n}^{\circ} \backslash P_{n}, N_{n}^{\circ} \backslash N_{n} ; s^{0}\right),
\end{aligned}
$$

where the left-hand side is our notation from Definition 5.1 and the right-hand side is the notation of [Hes92]. Since $\hat{M} \backslash \stackrel{B}{2}_{2} \subseteq \hat{M} \backslash B_{1}$, there is a restriction map $\Gamma\left(\hat{M}, B_{1}\right) \rightarrow \Gamma\left(\hat{M}, \stackrel{\circ}{B}_{2}\right)$, which is clearly a homotopy equivalence. The relative scanning map defined in [Hes92] (page 198) is a map

$$
\gamma: C\left(N_{n}, P_{n} ; s^{0}\right) \longrightarrow \Gamma\left(N_{n}^{\circ} \backslash P_{n}, N_{n}^{\circ} \backslash N_{n} ; s^{0}\right)
$$

which is a weak equivalence by the Proposition in $\S 2.3$ of [Hes92], since $\left(N_{n}, P_{n}\right)$ is connected. By inspecting the definition of $\gamma$ in [Hes92] and the definition of $\mathbf{s}$ at the beginning of this section, one sees that the following diagram commutes up to homotopy:

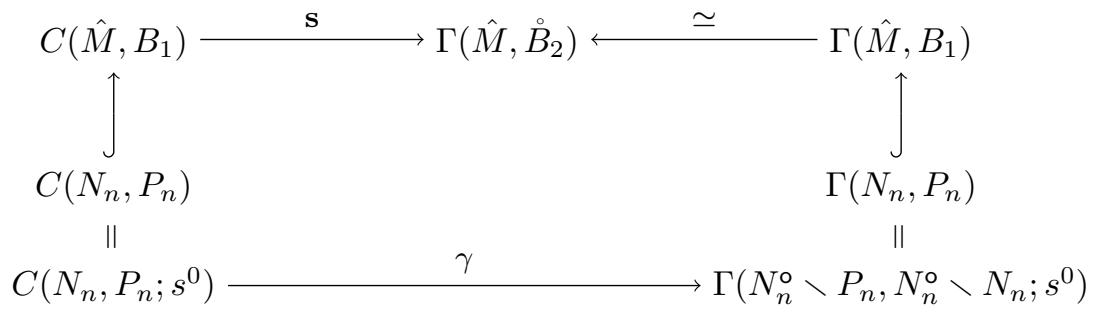

The spaces $C\left(N_{n}, P_{n}\right)$ form a filtration of $C\left(\hat{M}, B_{1}\right)$ so that every compact subset is contained in some finite stage of the filtration. The map $i: \operatorname{colim}_{n}\left(C\left(N_{n}, P_{n}\right)\right) \rightarrow$ $C\left(\hat{M}, B_{1}\right)$ is therefore a continuous bijection whose inverse is continuous on compact subsets - hence a weak equivalence. There is an analogous weak equivalence $j$ when $C$ is replaced by $\Gamma$. The map $\gamma$ respects these filtrations so it induces a map $\gamma_{\infty}: \operatorname{colim}_{n}\left(C\left(N_{n}, P_{n}\right)\right) \rightarrow \operatorname{colim}_{n}\left(\Gamma\left(N_{n}, P_{n}\right)\right)$ which is a weak equivalence since colim(-) commutes with $\pi_{*}(-)$. There is a commutative pentagon formed by $i, j, \gamma_{\infty}$, and the top two horizontal maps of the above square. We now deduce that $\mathbf{s}$ is a weak equivalence since the other four maps in the pentagon are weak equivalences.

This proves part (c) of Lemma 5.4, and therefore completes the proof of Theorem B. We can now apply this theorem together with homological stability for oriented configuration spaces to prove Theorem A for manifolds admitting boundary.

Corollary 5.9. If $M$ admits boundary, the lift $s^{+}: C_{k}^{+}(M, e) \rightarrow \Gamma_{k}^{+}(M, e)$ of the scanning map induces an isomorphism on $H_{*}(-; \mathbb{Z})$ in the range $* \leqslant(k-5) / 3$ and a surjection for $* \leqslant(k-2) / 3$.

Proof. We have a commutative square

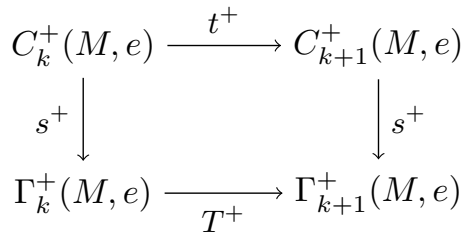


which double covers the square (3.10). This uses the facts that (a) the oriented configuration space $C_{k+1}^{+}(M, e)$ pulls back to the oriented configuration space $C_{k}^{+}(M, e)$ along the stabilization map $t$ and (b) the double cover $\Gamma_{k}^{+}(M, e)$ can be characterized by the property that it pulls back to $C_{k}^{+}(M, e)$ along the scanning map $s$. Taking mapping telescopes with respect to $t^{+}$and $T^{+}$, we get a map

$$
s^{+}: \operatorname{Tel}\left(C_{k}^{+}(M, e)\right) \longrightarrow \operatorname{Tel}\left(\Gamma_{k}^{+}(M, e)\right)
$$

which double-covers the map $s: \operatorname{Tel}\left(C_{k}(M, e)\right) \rightarrow \operatorname{Tel}\left(\Gamma_{k}(M, e)\right)$. By Theorem B the latter is an acyclic map, and therefore so is any homotopy pullback of it, including (5.5). In particular, (5.5) is an integral homology equivalence.

Now consider the commutative square

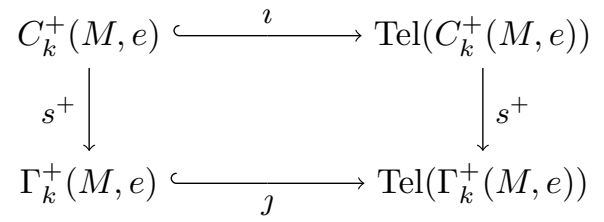

where $\imath$ and $\jmath$ are the evident inclusions into the mapping telescopes. Since the stabilization maps $T: \Gamma_{k}(M, e) \rightarrow \Gamma_{k+1}(M, e)$ are homotopy equivalences, so is the inclusion $\Gamma_{k}(M, e) \hookrightarrow \operatorname{Tel}\left(\Gamma_{k}(M, e)\right)$. Therefore, $\jmath$ is a weak equivalence, since it is a homotopy pullback of this inclusion.

So the bottom and right arrows in the above square are integral homology equivalences - hence $s^{+}: C_{k}^{+}(M, e) \rightarrow \Gamma_{k}^{+}(M, e)$ is an isomorphism on homology in the same range as $\imath$. Homological stability for oriented configuration spaces [Pal13] (Theorem 1.4 in the introduction) implies that $\imath$ is an isomorphism on homology in the claimed range.

\section{Closed manifolds}

In this section we describe how to extend Corollary 5.9 to the case of closed manifolds $M$, which finishes the proof of Theorem A. This is based on the arguments used by McDuff to prove Theorem 1.1 of [McD75]. Since the spaces $C_{k}^{+}(M)$ and $\Gamma_{k}^{+}(M)$ are both path-connected, the statement of Theorem $\mathrm{A}$ is true for $k \leqslant 4$, so we will now assume that $k \geqslant 5$.

Choose a Riemannian metric on $M$ and an isometric embedding $D \hookrightarrow M$ of the closed, $d$-dimensional unit disc $D$ (we may always scale the metric to make this possible). Following the ideas of $\S 6$ of [RW13] (see also $\S 5$ of [Pal13]), we define $U_{k}^{+}(M)$ to be the subspace of $C_{k}^{+}(M)$ of configurations which have a unique closest point in $D$ to the center $0 \in D$. The spaces $U_{k}^{+}(M)$ and $C_{k}^{+}(M \backslash\{0\})$ form an open cover of $C_{k}^{+}(M)$ so by excision the homotopy cofiber of the inclusion $C_{k}^{+}(M \backslash\{0\}) \hookrightarrow$ $C_{k}^{+}(M)$ is homology-equivalent to that of the inclusion $U_{k}^{+}(M \backslash\{0\}) \hookrightarrow U_{k}^{+}(M)$. For a configuration of $k \geqslant 3$ points with one marked point, giving an orientation of all $k$ points is equivalent to giving an orientation of the $k-1$ non-marked points. We are assuming that $k \geqslant 5$, so using this observation we see that the latter inclusion is 
homeomorphic to the inclusion $(D \backslash\{0\}) \times C_{k-1}^{+}(M \backslash\{0\}) \hookrightarrow D \times C_{k-1}^{+}(M \backslash\{0\})$, whose homotopy cofiber is $\Sigma^{d}\left(C_{k-1}^{+}(M \backslash\{0\})_{+}\right)$. So we have an identification:

$$
\operatorname{hocofib}\left(C_{k}^{+}(M \backslash\{0\}) \hookrightarrow C_{k}^{+}(M)\right) \simeq_{H \mathbb{Z}} \Sigma^{d}\left(C_{k-1}^{+}(M \backslash\{0\})_{+}\right),
$$

where $(-)_{+}$denotes adding an isolated basepoint and $\Sigma^{d}$ is the $d$ th reduced suspension.

We want to similarly identify the homotopy cofiber of the inclusion of section spaces $\Gamma_{k}^{+}(M \backslash D) \hookrightarrow \Gamma_{k}^{+}(M)$ with $\Sigma^{d}\left(\Gamma_{k}^{+}(M \backslash D)_{+}\right)$, up to homology equivalence. We can choose a $\mathrm{CW}$ structure on $M$ so that $(M, D)$ is a relative $\mathrm{CW}$-complex. Also, the subspace $M \backslash D$ is relatively compact (simply because $M$ is compact). Hence, applying Lemma 5.7, the restriction map $\Gamma(M) \rightarrow \Gamma(M, M \backslash D)=\Gamma(D)$ is a Serre fibration. Its fiber $F$ over the section-at-infinity in $\Gamma(D)$ is the space of all sections of $\dot{T} M \rightarrow M$ that are equal to $\infty$ on $D$. This contains a copy of $\Gamma(M \backslash D)$ as a subspace (extending $s \in \Gamma(M \backslash D)$ by $\infty$ on $D)$, and the inclusion of this subspace into $F$ is a homotopy equivalence (with homotopy inverse induced by any diffeotopy from the identity $M \rightarrow M$ to a diffeomorphism that takes $D$ onto a slightly larger disc).

The restriction of $\Gamma(M) \rightarrow \Gamma(D)$ to the path component $\Gamma_{k}(M)$ is also a Serre fibration, whose fiber we denote $F_{k}$. Similarly to the previous paragraph, this contains a homeomorphic copy of $\Gamma_{k}(M \backslash D)$, and the inclusion $\Gamma_{k}(M \backslash D) \hookrightarrow F_{k}$ is a homotopy equivalence.

The composition of $\Gamma_{k}(M) \rightarrow \Gamma(D)$ with the double cover $\Gamma_{k}^{+}(M) \rightarrow \Gamma_{k}(M)$ is again a Serre fibration, with fiber $F_{k}^{+}$over the section-at-infinity in $\Gamma(D)$, where $F_{k}^{+} \rightarrow F_{k}$ is the restriction of the double cover $\Gamma_{k}^{+}(M) \rightarrow \Gamma_{k}(M)$ to the subspace $F_{k} \subseteq$ $\Gamma_{k}(M)$. The further restriction of this double cover to $\Gamma_{k}(M \backslash D) \subseteq F_{k}$ is $\Gamma_{k}^{+}(M \backslash$ $D),{ }^{10}$ so we have an inclusion $\Gamma_{k}^{+}(M \backslash D) \hookrightarrow F_{k}^{+}$of double covers over the inclusion $\Gamma_{k}(M \backslash D) \hookrightarrow F_{k}$. The latter inclusion is a homotopy equivalence, and therefore the inclusion $\Gamma_{k}^{+}(M \backslash D) \hookrightarrow F_{k}^{+}$is a weak equivalence.

Note that $\Gamma(D) \cong \operatorname{Map}\left(D, S^{d}\right) \simeq S^{d}$. Applying the following general lemma to the Serre fibration $\Gamma_{k}^{+}(M) \rightarrow \Gamma(D)$ gives us an identification:

$$
\operatorname{hocofib}\left(\Gamma_{k}^{+}(M \backslash D) \hookrightarrow \Gamma_{k}^{+}(M)\right) \simeq_{H \mathbb{Z}} \quad \Sigma^{d}\left(\Gamma_{k}^{+}(M \backslash D)_{+}\right) .
$$

Lemma 6.1. Let $\pi: Y \rightarrow X$ be a Serre fibration with $X \simeq S^{d}$, and let $F=\pi^{-1}\left(x_{0}\right)$ for a point $x_{0} \in X$. Then the homotopy cofiber of the inclusion $F \hookrightarrow Y$ is homology equivalent to $\Sigma^{d}\left(F_{+}\right)$.

Proof. Choose a homotopy equivalence $X \rightarrow S^{d}$, and replace the composition $Y \rightarrow$ $X \rightarrow S^{d}$ by a fibration $p: Z \rightarrow S^{d}$ :

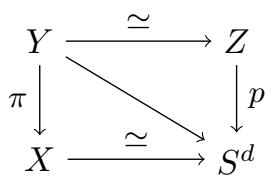

\footnotetext{
${ }^{10}$ One can see this from the characterization of $\Gamma_{k}^{+}(M)$ in the introduction as the double cover that pulls back to the oriented configuration space $C_{k}^{+}(M)$ along the scanning map $C_{k}(M) \rightarrow$ $\Gamma_{k}(M)$, together with the fact that $C_{k}^{+}(M)$ pulls back along the inclusion $C_{k}(M \backslash D) \hookrightarrow C_{k}(M)$ to $C_{k}^{+}(M \backslash D)$.
} 
Denote the image of $x_{0}$ in $S^{d}$ by $y_{0}$, and denote the fiber $p^{-1}\left(y_{0}\right)$ by $F^{\prime}$. The homology of the homotopy cofiber of $F \hookrightarrow Y$ is the homology of the pair $(Y, F)$. Using the long exact sequence on homotopy groups for the map of fibrations (6.3) and the long exact sequence on homology for the map of pairs $(Y, F) \rightarrow\left(Z, F^{\prime}\right)$, we have $H_{*}(Y, F) \cong H_{*}\left(Z, F^{\prime}\right)$. Let $D_{+}$and $D_{-}$be complementary hemispheres of $S^{d}$, so $\partial D_{+}=\partial D_{-}=S^{d-1}$, and say $y_{0}$ is in the interior of $D_{-}$. The inclusion $F^{\prime} \hookrightarrow p^{-1}\left(D_{-}\right)$is a homotopy equivalence, so $H_{*}\left(Z, F^{\prime}\right) \cong H_{*}\left(Z, p^{-1}\left(D_{-}\right)\right)$. By excision, this is isomorphic to $H_{*}\left(p^{-1}\left(D_{+}\right), p^{-1}\left(S^{d-1}\right)\right)$. Since $D_{+}$is contractible, the restriction of the fibration $p$ over $D_{+}$is homotopy equivalent (over $D_{+}$) to the trivial fibration $D_{+} \times F^{\prime} \rightarrow D_{+}$, and so this homology group is, in turn, isomorphic to $H_{*}\left(D_{+} \times F^{\prime}, S^{d-1} \times F^{\prime}\right)$. The inclusion $S^{d-1} \times F^{\prime} \hookrightarrow D_{+} \times F^{\prime}$ is a cofibration, so this is the reduced homology of the quotient, which is homeomorphic to $\Sigma^{d}\left(F_{+}^{\prime}\right)$. Finally, $F \simeq F^{\prime}$, so this is $\widetilde{H}_{*}\left(\Sigma^{d}\left(F_{+}\right)\right)$. We therefore have the required isomorphism of homology groups. Moreover, the zig-zag

$$
\begin{aligned}
(Y, F) \stackrel{\simeq_{H \mathbb{Z}}}{\longrightarrow}\left(Z, F^{\prime}\right) \stackrel{\simeq_{\mathrm{w}}}{\longrightarrow}\left(Z, p^{-1}\left(D_{-}\right) \stackrel{\simeq_{H \mathbb{Z}}}{\longleftarrow}\left(p^{-1}\left(D_{+}\right), p^{-1}\left(S^{d-1}\right)\right)\right. \\
\left(D_{+}, S^{d-1}\right) \times F \stackrel{\simeq_{\mathrm{w}}}{\longrightarrow}\left(D_{+}, S^{d-1}\right) \times F^{\prime}
\end{aligned}
$$

induces a homology equivalence between $(Y, F)$ and $\left(D^{d}, S^{d-1}\right) \times F=\Sigma^{d}\left(F_{+}\right)$.

With this preliminary work done, we can now easily deduce deduce Theorem A for closed manifolds from Theorem A for manifolds admitting boundary, which was Corollary 5.9.

Proof of Theorem A for closed manifolds. Let $\hat{D}$ be another closed disc in $M$, containing $D$, which is sufficiently larger than $D$ so that for configurations $c$ in $M \backslash \hat{D}$ the section $s(c)$ is equal to $\infty$ on an open neighborhood of $D$. This means that the scanning map is a map of pairs

$$
s:\left(C_{k}(M), C_{k}(M \backslash \hat{D})\right) \longrightarrow\left(\Gamma_{k}(M), \Gamma_{k}(M \backslash D)\right),
$$

and it therefore also lifts to a map of pairs

$$
s^{+}:\left(C_{k}^{+}(M), C_{k}^{+}(M \backslash \hat{D})\right) \longrightarrow\left(\Gamma_{k}^{+}(M), \Gamma_{k}^{+}(M \backslash D)\right) .
$$

Using the identifications (6.1) and (6.2) above, and the fact that the two inclusions $C_{k}^{+}(M \backslash \hat{D}) \hookrightarrow C_{k}^{+}(M \backslash D) \hookrightarrow C_{k}^{+}(M \backslash\{0\})$ are homotopy equivalences, we obtain a map of long exact sequences:

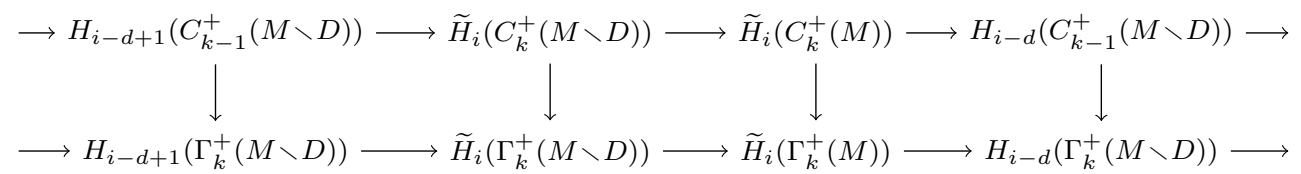

The middle two maps are induced by the scanning map $s^{+}$, and, following through the identifications of the homotopy cofibers above, one sees that the outer vertical maps are induced by the composition $s^{+} \circ t^{+}$of the stabilization map followed by the scanning map. 
Since $M \backslash D$ admits boundary $\partial D$, we can apply Theorem 1.4 and Corollary 5.9 to conclude that the first, second, fourth, and fifth (not drawn) vertical maps above are isomorphisms in the ranges $3 i \leqslant k+3 d-9,3 i \leqslant k-5,3 i \leqslant k+3 d-6$, and $3 i \leqslant$ $k-2$, respectively. Since $d \geqslant 2$, these conditions all hold when $3 i \leqslant k-5$, so by the five-lemma the third vertical map above is an isomorphism in this range. Moreover, in the range $3 i \leqslant k-2$ the second vertical map is a surjection and the fourth and fifth vertical maps are isomorphisms, which implies that the third vertical map is a surjection.

Using Theorem A, we can now prove a similar result regarding the scanning map $C_{k}(M) \rightarrow \Gamma_{k}(M)$ on twisted homology $H_{*}\left(-; \mathbb{Z}^{(-1)}\right)$. Recall that $R^{(-1)}$, for a ring $R$, is the $R[\mathbb{Z} / 2]$-module where the generator of $\mathbb{Z} / 2$ acts by multiplication by -1 . The fundamental groups $\pi_{1}\left(C_{k}(M)\right)$ and $\pi_{1}\left(\Gamma_{k}(M)\right)$ have natural maps to $\mathbb{Z} / 2$, described in $\S 3.6$, so $R^{(-1)}$ becomes a module over their group-rings, too. See the introduction or [BCT89] for a discussion of the relationship between $H_{*}\left(C_{k}(M) ; \mathbb{Z}^{(-1)}\right)$ and the homology of the spaces appearing in the generalized Snaith splitting introduced in [Böd87].

Corollary 6.2. The homomorphism $H_{*}\left(C_{k}(M) ; \mathbb{Z}^{(-1)}\right) \rightarrow H_{*}\left(\Gamma_{k}(M) ; \mathbb{Z}^{(-1)}\right)$ induced by the scanning map is an isomorphism for $* \leqslant(k-5) / 3$ and a surjection for $* \leqslant$ $(k-2) / 3$.

Proof. There is a short exact sequence of $\mathbb{Z}[\mathbb{Z} / 2]$-modules

$$
0 \rightarrow \mathbb{Z} \rightarrow \mathbb{Z}[\mathbb{Z} / 2] \rightarrow \mathbb{Z}^{(-1)} \rightarrow 0
$$

which, via the homomorphism $\pi_{1}\left(C_{k}(M)\right) \rightarrow \mathbb{Z} / 2$, can also be viewed as a short exact sequence of $\mathbb{Z}\left[\pi_{1}\left(C_{k}(M)\right)\right]$-modules, so we obtain a long exact sequence of the homology groups of $C_{k}(M)$ with these coefficients. We can also do the same for $\Gamma_{k}(M)$ using the homomorphism $\pi_{1}\left(\Gamma_{k}(M)\right) \rightarrow \mathbb{Z} / 2$ instead. Since the homomorphism $\pi_{1}\left(C_{k}(M)\right) \rightarrow \mathbb{Z} / 2$ factors through this one via the scanning map, it induces a map between these long exact sequences. Identifying $H_{i}\left(C_{k}(M) ; \mathbb{Z}[\mathbb{Z} / 2]\right)$ with $H_{i}\left(C_{k}^{+}(M) ; \mathbb{Z}\right)$ and $H_{i}\left(\Gamma_{k}(M) ; \mathbb{Z}[\mathbb{Z} / 2]\right)$ with $H_{i}\left(\Gamma_{k}^{+}(M) ; \mathbb{Z}\right)$, and abbreviating $H_{i}(-)=$ $H_{i}(-; \mathbb{Z})$, this map of long exact sequences is

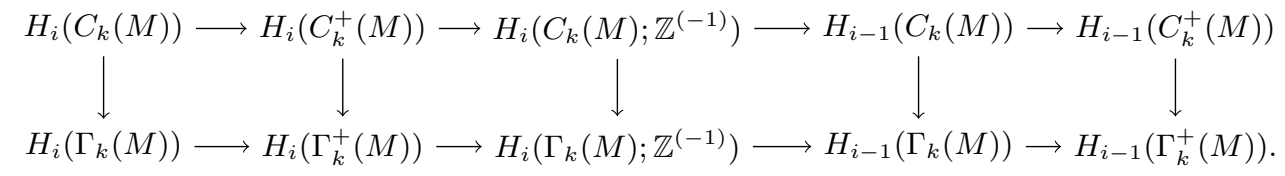

In the range $3 i \leqslant k-5$ the first, second, fourth, and fifth vertical maps above are isomorphisms, by Theorems 1.3 and $\mathrm{A}$, so by the five-lemma the middle vertical map is also an isomorphism. In the range $3 i \leqslant k-2$ the second, fourth, and fifth vertical maps are isomorphisms, so the middle vertical map is a surjection.

\section{References}

[BCT89] C.-F. Bödigheimer, F. Cohen, and L. Taylor. On the homology of configuration spaces. Topology 28.1 (1989), pp. 111-123. 
[Ber82] A.J. Berrick. An approach to algebraic K-theory. Vol. 56. Research Notes in Mathematics. Boston, Mass.: Pitman (Advanced Publishing Program), 1982.

[BM14] Martin Bendersky and Jeremy Miller. Localization and homological stability of configuration spaces. Q. J. Math. 65.3 (2014), pp. 807-815.

[Böd87] C.-F. Bödigheimer. Stable splittings of mapping spaces. In Algebraic topology (Seattle, Wash., 1985) Lecture Notes in Math. 1286 Springer Verlag, Berlin (1987), pp. 174-187.

[Cha80] R.M. Charney. Homology stability for $\mathrm{GL}_{n}$ of a Dedekind domain. Invent. Math. 56.1 (1980), pp. $1-17$.

[Chu12] T. Church. Homological stability for configuration spaces of manifolds. Invent. Math. 188.2 (2012), pp. 465-504.

[CP14] F. Cantero and M. Palmer. On homological stability for configuration spaces on closed background manifolds. ArXiv: 1406.4916v2. 2014.

[FVB62] E. Fadell and J. Van Buskirk. The braid groups of $E^{2}$ and $S^{2}$. Duke Math. J. 29 (1962), pp. 243-257.

[Gal11] S. Galatius. Stable homology of automorphism groups of free groups. Ann. of Math. (2) 173.2 (2011), pp. 705-768.

[GKY] M.A. Guest, A. Kozlowski, and K. Yamaguchi. Stability of configuration spaces of positive and negative particles. Unpublished preprint; available online at http://www.mimuw.edu.pl/ akoz/topology/GKYPDF/GKY5.pdf

[Har85] J.L. Harer. Stability of the homology of the mapping class groups of orientable surfaces. Ann. of Math. (2) 121.2 (1985), pp. 215-249.

[Hat95] A. Hatcher. Homological stability for automorphism groups of free groups. Comment. Math. Helv. 70.1 (1995), pp. 39-62.

[Hes92] L. Hesselholt. A homotopy theoretical derivation of $Q \operatorname{Map}(K,-)_{+}$. Math. Scand. 70.2 (1992), pp. 193-203.

[HV98] A. Hatcher and K. Vogtmann. Cerf theory for graphs. J. London Math. Soc. (2) 58.3 (1998), pp. 633-655.

[HW10] A. Hatcher and N. Wahl. Stabilization for mapping class groups of 3-manifolds. Duke Math. J. 155.2 (2010), pp. 205-269.

[Lur09] J. Lurie. Derived Algebraic Geometry VI: $\mathbb{E}[k]$-Algebras. ArXiv: 0911.0018v1. 2009.

[McD75] D. McDuff. Configuration spaces of positive and negative particles. Topology 14 (1975), pp. 91-107.

[ML88] R.J. Milgram and P. Löffler. The structure of deleted symmetric products. In: Braids (Santa Cruz, CA, 1986) Contemp. Math. 78 Amer. Math. Soc., Providence, RI (1988), pp. 415-424.

[MP14] J. Miller and M. Palmer. A twisted homology fibration criterion and the twisted group-completion theorem. ArXiv:1409.4389v1. 2014.

[MS75] D. McDuff and G. Segal. Homology fibrations and the "group-completion" theorem. Invent. Math. 31.3 (1975/76), pp. 279-284.

[MW07] I. Madsen and M. Weiss. The stable moduli space of Riemann surfaces: Mumford's conjecture. Ann. of Math. (2) 165.3 (2007), pp. 843-941.

[Pal13] M. Palmer. Homological stability for oriented configuration spaces. Trans. Amer. Math. Soc. 365.7 (2013), pp. 3675-3711.

[Qui73] D. Quillen. Finite generation of the groups $K_{i}$ of rings of algebraic integers. In: Algebraic K-theory, I: Higher K-theories (Proc. Conf., Battelle Memorial Inst., Seattle, Wash., 1972). Lecture Notes in Math., Vol. 341, Springer-Verlag, Berlin (1973), pp. 179-198. .

[RW13] O. Randal-Williams. 'Group-completion', local coefficient systems and perfection. Q. J. Math. 64.3 (2013), pp. 795-803. 
[RW13] O. Randal-Williams. Homological stability for unordered configuration spaces. $Q$. J. Math. 64.1 (2013), pp. 303-326.

[Sal01] P. Salvatore. Configuration spaces with summable labels. In: Cohomological methods in homotopy theory (Bellaterra, 1998). Progr. Math. 196 Birkhäuser, Basel (2001), pp. 375-395.

[Seg79] G. Segal. The topology of spaces of rational functions. Acta Math. 143.1-2 (1979), pp. 39-72.

[Sna74] V.P. Snaith. A stable decomposition of $\Omega^{n} S^{n} X$. J. London Math. Soc. (2) 7 (1974), pp. 577-583.

[Wah08] N. Wahl. Homological stability for the mapping class groups of non-orientable surfaces. Invent. Math. 171.2 (2008), pp. 389-424.

Jeremy Miller jkmiller@stanford.edu

Department of Mathematics, Stanford University, Building 380, Stanford, California, 94305, USA

Martin Palmer mpalm_01@uni-muenster.de

Mathematisches Institut, WWU Münster, Einsteinstraße 62, 48149 Münster, Germany 